\title{
Numerical simulation of the transient hydrogen trapping process using an analytical approximation of the McNabb and Foster equation. Part 2: domain of validity
}

Yann CHARLES ${ }^{1}$, Sofiane BENANNOUNE, Jonathan MOUGENOT and Monique GASPERINI

Université Sorbonne Paris Nord, Laboratoire des Sciences des Procédés et des Matériaux, LSPM, CNRS, UPR 3407, F-93430, Villetaneuse, France

\begin{abstract}
In a previous study [1], a resolution scheme called Generalized Oriani's Approximation (GOA) was proposed to solve a transient transport and trapping problem in the Abaqus Finite Element software. This proposition was motivated by the convergence of the Finite Element problem linked to the estimation of several functions during the computation (and especially the $d u d t$ one). In this study, the GOA is shown to be able to provide an accurate estimation of the trapped concentration in transient trapping processes as soon as the time increment is small enough for two configurations: hydrogen in metals and water in polymers. An estimation of the induced error is given. The GOA approach is illustrated on a simple configuration with various trapping parameters. Last, the ability of Abaqus to converge while modeling a transient trapping and transport problem is analyzed considering several $d u d t$ : it is shown especially that its estimation based on the GOA allows the solver to efficiently converge toward the solution.
\end{abstract}

keywords: Finite Element, Diffusion, Kinetic trapping, Abaqus, User Subroutines

\section{Introduction}

The improvement of the life-time estimation of in-service structures leads to the improvement of the numerical evaluation of several parameters or phenomena. One of these phenomena is related to the penetration of impurities into the material, which diffuse and interact with the materials (as hydrogen in metals, or water in polymers). These interactions can affect the mechanical behavior [2-5] or the fracture resistance [6,7]; they can also induce new phases [8], damage [9-14] and internal stress [15-18]. Moreover, materials characteristic act on diffusion due to the presence of traps for impurities which can be either defects (grain boundaries, dislocations, vacancies...) [19,20] or local specific chemical processes [21,22].

Trapping can be described using differential equations, involving the trapped and diffusive concentrations, material parameters, kinetic constants and trap and diffusion sites densities [2325]. For problems dealing with hydrogen diffusion and trapping in metals, such an approach is usually replaced by a simpler expression expressed by Oriani [26]. This approximation is widely used (see among other [9,27-33]) and allows an efficient Finite Element (FE) implementation using coupled displacement and diffusion approaches.

\footnotetext{
${ }^{1}$ Corresponding author, yann.charles@univ-paris13.fr, tel: (33) 1494034 61, fax: (33) 149403938
} 
In specific applications, e.g. for cases in which cycling hydrogen expositions occurs [34], dealing with the differential equation instead of any kind of long-term approximation is mandatory. In this case, the FE implementation has to be reinvestigated and at each resolution step, the resolution scheme employed must be robust enough in order to get reliable results. For Abaqus, extra functions (and especially the $d u d t$ one, see section 2) needed for the convergence of the global FE problem, must be provided leading to complex calculations or inaccurate results.

In the frame of hydrogen-material interactions, such a scheme has been proposed to solve the McNabb \& Foster trapping equation [23] and to provide Abaqus these extra needed functions [1]. This scheme (named thereafter "Generalized Oriani's Approximation" or GOA) has been successfully compared to results obtained from experiments or computations [1] and successfully applied to several configurations in terms of geometries, mechanical behaviors and boundary conditions [34-40].

However, the domain of validity of this approach has not been determined, especially for the Abaqus implementation, which is the prior justification of the GOA development. The aims of the present paper are thus:

- to investigate the ability of the GOA to provide an accurate approximation of the exact solution of trapping differential equations;

- to investigate the importance of the $d u d t$ parameter in the Abaqus implementation for the software convergence and to evaluate the estimations of this parameter based on the GOA.

Two trapping configurations are considered: hydrogen in metals and water in polymers for which the trapping differential equations are very similar.

The paper is organized as follow.

The problem fundamentals are first recalled: diffusion and trapping differential equations, their implementation in Abaqus and the GOA formulation (including its implementation scheme).

Then, an upper bound of the error induced by the GOA on the trapped concentration evaluation is derived to estimate its ability to provide an accurate enough estimation of the trapping differential equation solution.

An illustration of this ability is made for a very specific system.

Last, the Abaqus implementation is focused on the role of the dudt function in the global software convergence and its evaluation by the GOA.

In the following, ' $\Delta$ ' and ' $\delta$ ' denote an increment of a given variable or function, and ' $\boldsymbol{\nabla}$ ' the Nabla operator. Vector and matrix are written in bold scripts.

\section{Implementation of a transport and transient trapping problem in Abaqus}

In the following, two configurations will be focused on:

- trapping of hydrogen atoms in polycrystalline metallic materials;

- bounding of water molecules in polymer materials (like the epoxy resin).

Other configurations could have been considered using the same kind of differential equation, but for the sake of simplicity, it has been limited to these two. Water molecules and hydrogen atoms are thereafter refered as "impurities" and the retention process as "trapping". For these 
two problems, $C_{L}$ denotes the concentration of mobile impurities (water or hydrogen), and $C_{T}$ the concentration of the non-mobile (or trapped) ones.

The mobile particle flux is expressed as (assuming a stress-free configuration [41-43])

$$
\boldsymbol{\varphi}=-D_{L} \boldsymbol{\nabla} C_{L}
$$

where $D_{L}$ is the diffusion coefficient $\left(\mathrm{m}^{2} / \mathrm{s}\right)$. The global diffusion and trapping process is deduced from equation (2.1) based on the Fick law

$$
\frac{\partial C_{L}}{\partial t}+\frac{\partial C_{T}}{\partial t}-D_{L} \nabla \cdot\left(\nabla C_{L}\right)=0
$$

\subsection{Transient trapping}

For hydrogen diffusion in metals, $\partial C_{T} / \partial t$ is computed using the McNabb \& Foster equation [23]

$$
\frac{\partial C_{T}}{\partial t}=k W C_{L}\left(1-\frac{C_{T}}{N_{T}}\right)-p C_{T} \text { with } \frac{C_{L}}{N_{L}} \ll 1
$$

where $W=N_{T} / N_{L}, N_{L}$ being the diffusion site density and $N_{T}$ the trapping one (assumed in the following to be both constant in time and space).

In the context of a diffusion and trapping of water in polymers, $\partial C_{T} / \partial t$ is set following the Carter \& Kibler's equation [24]

$$
\frac{\partial C_{T}}{\partial t}=k W C_{L}-p C_{T}
$$

Both equations corresponds to first order kinetic equation [44], as expressed for hydrogen in [45]; $k$ and $p$ are constant and positive material parameters which represent respectively the trapping and detrapping reaction rate constants, with, e.g., for hydrogen $[1,46]$

$$
\left\{\begin{array}{c}
p=v_{0} e^{-E_{d} / k T} \\
k=D_{L} / \lambda^{2}
\end{array}\right.
$$

$E_{d}$ is the trapping energy, $\lambda$ represents the distance between two interstitial sites, and $v_{0}$ the hydrogen atom jump attempt frequency. $k$ and $T$ are respectively the Boltzmann constant and the temperature.

Equation (2.4) can be seen also a particular case of equation (2.2), for which $C_{T} / N_{T} \ll 1, \forall C_{T}$, i.e., for which $C_{T}$ is not bounded (or for which $N_{T} \rightarrow+\infty$ ).

Let's introduced $\theta_{\alpha / \alpha \in\{L, T\}} \in[0,1]$, the diffusion or trapping site occupancy, so that $C_{\alpha}=$ $N_{\alpha} \theta_{\alpha}$. Equation (2.3) and (2.4) can thus be rewritten as 


$$
\left\{\begin{array}{c}
(a) \frac{\partial C_{L}}{\partial t}+N_{T} \frac{\partial \theta_{T}}{\partial t}-D_{L} \boldsymbol{\nabla} \cdot\left(\nabla C_{L}\right)=0 \\
(b 1) \frac{\partial \theta_{T}}{\partial t}=k \theta_{L}\left(1-\theta_{T}\right)-p \theta_{T}(\text { Mc Nabb and Foster equation }) \\
\text { (b2) } \frac{\partial \theta_{T}}{\partial t}=k \theta_{L}-p \theta_{T} \text { (Carter and Kibler equation) }
\end{array}\right.
$$

While dealing with a coupled diffusion and trapping problem, the differential equation set (ab) has to be solved along with any other relevant problems (mechanical ...). In the context of hydrogen trapping, $\theta_{T}$ is classically approximated by its steady state solution $\theta_{T}^{s t, O r i}$ as proposed by Oriani for equation (2.6)b1 [26]; the corresponding expression for equation (2.6)b2 can either be deduced from Oriani's one or from [24]

$$
\left\{\begin{array}{c}
(b 1) \theta_{T}^{\text {st,ori }}=\frac{k \theta_{L}}{p+k \theta_{L}} \\
\text { (b2) } \theta_{T}^{\text {st,ori }}=\frac{k}{p} \theta_{L}
\end{array}\right.
$$

It is worth noting that these two solutions are equivalent for $k \theta_{L} \ll p$. The resolution of the transient trapping equations (2.6)b1 and $\mathrm{b} 2$ is more challenging, especially when commercial simulation software is used. In the following, we present the strategy used in Abaqus.

\subsection{Implementation in Abaqus}

The resolution of a coupled mechanical-impurities transport and trapping problem in Abaqus is made using a UMATHT subroutine, written in Fortran [47], in the frame of a 'coupled tempdisp' resolution scheme (in such a case, the nodal degree of freedom $\mathrm{n}^{\circ} 11$ is considered to be the mobile impurity concentration $C_{L}$ ).

The UMATHT subroutine is called by Abaqus at each mesh integration points and for all Newton-Raphson iterations. The software provides a set of variables to the subroutines, which compute in return all the needed values to complete the current Newton-Raphson loop; and thus provide the local contribution to the force residuals vector and to its Jacobian matrix. This process is summarized on Figure 1.

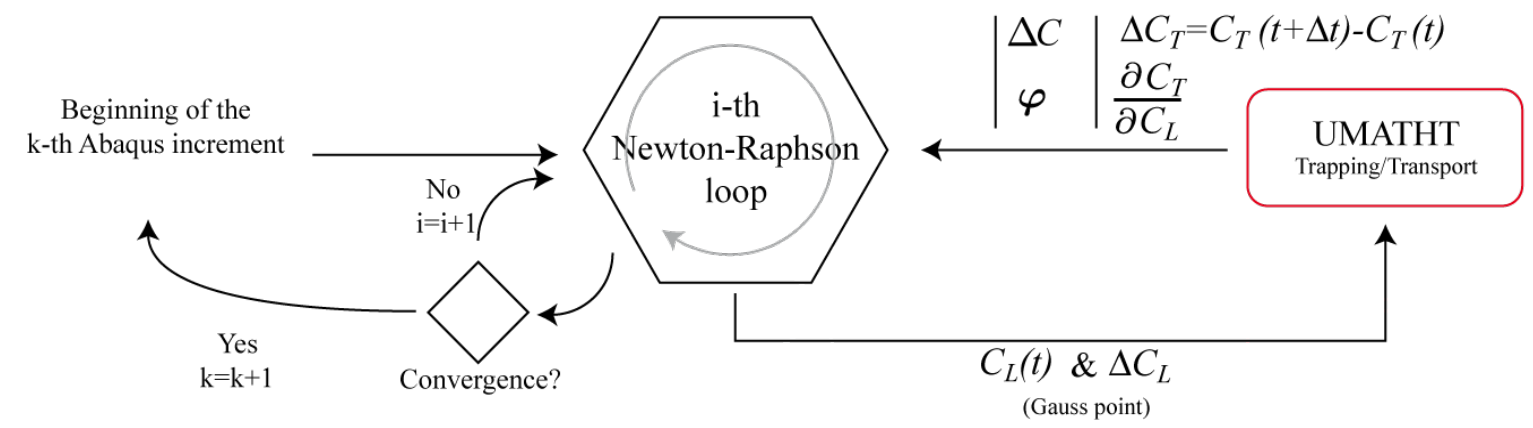

Figure 1. Flowshart of the UMATHT subroutine intercations with Abaqus software.

In the case on a coupled transport and trapping problem (see [27,29] for the correspondence between a thermal and a diffusion approach), the total concentration impurity $C$ can be set as: 


$$
C=C_{L}+C_{T}
$$

where $C_{L}$ is the mobile particles concentration and $C_{T}$ the trapped one. For Abaqus, $C_{L}$ is a degree of freedom of the Finite Element problem $\left(\mathrm{n}^{\circ} 11\right)$ while $C_{T}$ is an internal variable used in the UMATHT subroutine, defined and computed at each Gauss point.

During the computation, Abaqus provides the UMATHT with the following values (the name of which being the same than in the subroutine):

- temp: the diffusive concentration $C_{L}$ at the beginning of the current increment (which is named kinc in UMATHT subroutine);

- dtemp: the current diffusive concentration increment $\Delta C_{L}$ after several NewtonRaphson loops;

while must be computed, among other:

- $u$ : the total concentration $C$ at the end of the current increment $\left(C=C_{L}+\Delta C_{L}+C_{T}+\right.$ $\Delta C_{T}$ ). Especially, $\Delta C_{T}$ has to be computed;

- $d u d t$ : corresponds to $\partial C / \partial C_{L}=1+\partial C_{T} / \partial C_{L}$;

- flux: the $C_{L}$ flux (equation (2.1)).

Among the above quantities, it is mandatory to get a converged Abaqus solution, to:

1. compute the temporal evolution of $C_{T}$;

2. express an estimation of $\partial C_{T} / \partial C_{L}$ (i.e., of $d u d t$ ).

In the case of hydrogen in metallic material when an instantaneous trapping is assumed, a simple relationship exists between $C_{L}$ and $C_{T}$ (based on Oriani's work [26]) from which $C_{T}$ and $\partial C_{T} / \partial C_{L}$ can be easily deduced [48,49]. When dealing with a transient trapping, the temporal evolution of $C_{T}$ is defined by a differential equation (see equation (2.6)), depending on the diffusive concentration value $C_{L}$. While $C_{T}$ temporal evolution can be determined based on any numerical resolution scheme (using e.g. a $\theta$-method [50]), the evaluation of $\partial C_{T} / \partial C_{L}$ is a more complex problem, though being the key point for a robust implementation in Abaqus, allowing the code to produce accurate $C_{L}$ and $C_{T}$ spatio-temporal evolutions. The evaluation of $\partial C_{T} / \partial C_{L}$ has been our main concern while proposing an analytical approximation of the McNabb \& Foster kinetic trapping equation.

\subsection{GOA formulation \& introduction in the UMATHT subroutine}

The GOA's underlying assumption is that between two time increments $t$ and $t+\Delta t, \theta_{L}$ is constant and equal to $\theta_{L}(t+\Delta t)$; with such an assumption, the trapping differential equations (equations (2.6)b1 and $\mathrm{b} 2$ ) are solvable between $t$ and $t+\Delta t$ [1]. The GOA has been implemented using an incremental scheme, as detailed below, to be able to capture the influence of the temporal evolutions of $\theta_{L}$.

\section{Hydrogen in metallic materials}

Following the GOA assumption, Equation (2.6)b1 can be solved as [1]

$$
\theta_{T}(t+\Delta t)=\left[\theta_{T}(t)-\frac{k \theta_{L}(t+\Delta t)}{k \theta_{L}(t+\Delta t)+p}\right] e^{-\left(k \theta_{L}(t+\Delta t)+p\right) \Delta t}+\frac{k \theta_{L}(t+\Delta t)}{k \theta_{L}(t+\Delta t)+p}
$$

The $\Delta \theta_{T}$ increment is thus 


$$
\Delta \theta_{T}=\theta_{T}(t+\Delta t)-\theta_{T}(t)=\left(e^{-\left(k+p \theta_{L}(t+\Delta t)\right) \Delta t}-1\right)\left[\theta_{T}(t)-\frac{k \theta_{L}(t+\Delta t)}{k \theta_{L}(t+\Delta t)+p}\right]
$$

The total hydrogen concentration increment can thus be deduced from equation (2.10)

$$
\Delta C=\Delta C_{L}+\Delta C_{T}=N_{L} \Delta \theta_{L}+N_{T} \Delta \theta_{T}
$$

and

$$
\Delta C=\Delta C_{L}+\Delta C_{T}=\Delta C_{L}+\left.\frac{\partial C_{T}}{\partial C_{L}}\right|_{t} \Delta C_{L}+\left.\frac{\partial C_{T}}{\partial t}\right|_{C_{L}} \Delta t=(\text { dudt }) \Delta C_{L}+\left.\frac{\partial C_{T}}{\partial t}\right|_{C_{L}} \Delta t
$$

with $C_{T}=C_{T}\left(C_{L}(t), t\right)$. In equation (2.12), $d u d t$ is so that

$$
d u d t=1+\left.\frac{\partial C_{T}}{\partial C_{L}}\right|_{t}=1+\left.W \frac{\partial \theta_{T}}{\partial \theta_{L}}\right|_{t}
$$

with

$$
\begin{gathered}
\left.\frac{\partial \theta_{T}}{\partial \theta_{L}}\right|_{t} \\
=-k \Delta t\left[\theta_{T}(t)-\frac{k \theta_{L}(t+\Delta t)}{k \theta_{L}(t+\Delta t)+p}\right] e^{-\left(k \theta_{L}(t+\Delta t)+p\right) \Delta t} \\
+\frac{k p}{\left(k \theta_{L}(t+\Delta t)+p\right)^{2}}\left(1-e^{-\left(k \theta_{L}(t+\Delta t)+p\right) \Delta t}\right)
\end{gathered}
$$

For an instantaneous trapping [26], equation (2.12) becomes, consistently with [27],

$$
\Delta C=\Delta C_{L}+\Delta C_{T}=\Delta C_{L}+\left.\frac{\partial C_{T}}{\partial C_{L}}\right|_{t} \Delta C_{L}=(d u d t) \Delta C_{L}
$$

with $C_{T}=C_{T}\left(C_{L}(t)\right)$, leading to [48]

$$
d u d t=\frac{C_{L}+C_{T}\left(1-\theta_{T}\right)}{C_{L}}
$$

The incremental implementation is made using equation (2.10) for $\Delta C_{T}$, while $d u d t$ is given by equation (2.14).

2. Water in polymer materials

The same approach is applied on Equation (2.6)b2, yielding

$$
\theta_{T}(t+\Delta t)=\left[\theta_{T}(t)-\frac{k}{p} \theta_{L}(t+\Delta t)\right] e^{-p \Delta t}+\frac{k}{p} \theta_{L}(t+\Delta t)
$$

leading to

$$
\Delta \theta_{T}=\theta_{T}(t+\Delta t)-\theta_{T}(t)=\left(e^{-k \Delta t}-1\right)\left[\theta_{T}(t)-\frac{k}{p} \theta_{L}(t+\Delta t)\right]
$$

The total water concentration increment can thus be deduced as

and

$$
\Delta C=\Delta C_{L}+\Delta C_{T}=N_{L} \Delta \theta_{L}+N_{T} \Delta \theta_{T}
$$




$$
\Delta C=\Delta C_{L}+\Delta C_{T}=\Delta C_{L}+\left.\frac{\partial C_{T}}{\partial C_{L}}\right|_{t} \Delta C_{L}+\left.\frac{\partial C_{T}}{\partial t}\right|_{C_{L}} \Delta t=(d u d t) \Delta C_{L}+\left.\frac{\partial C_{T}}{\partial t}\right|_{C_{L}} \Delta t
$$

with $C_{T}=C_{T}\left(C_{L}(t), t\right)$ and

$$
d u d t=1+W \frac{k}{p}\left(1-e^{-p \Delta t}\right)
$$

It is worth noting that, in this case, the value of $d u d t$ as computed in the frame of the GOA is exact. From equation (2.6)b2, we can write

$$
\frac{\partial^{2} \theta_{T}}{\partial t \partial \theta_{L}}=k-p \frac{\partial \theta_{T}}{\partial \theta_{L}}
$$

or introducing $\chi=\partial \theta_{T} / \partial \theta_{L}$,

$$
\dot{\chi}=k-p \chi
$$

This equation can be solved as follows, assuming that $\chi=0$ for $\Delta t=0$,

$$
\chi=\frac{\partial \theta_{T}}{\partial \theta_{L}}(\Delta t)=\frac{k}{p}\left(1-e^{-p \Delta t}\right)
$$

The incremental solution for $d u d t$ (equation (2.21)) consequently corresponds to the theoretical value at the time $\Delta t$. Such a result indicates that $d u d t$, at each time increment, does not depend on its previous values. For a constant time increment $\Delta t$ and because of the linearity of the differential equation (2.6)b2, $d u d t$ remains constant during the whole computation and is equal to $1+W \frac{k}{p}\left(1-e^{-p \Delta t}\right)$ whatever $t$. This can be understood because equation (2.6)b2 corresponds to an unbounded trapping process $\left(\theta_{T} \ll 1\right)$ : at a given time, $d u d t$ describes the extra variation of $C_{T}, \delta C_{T}$, that have arisen due to an extra $C_{L}$ increment, $\delta C_{L}$, i.e,

$$
\text { for any } \Delta t,\left\{\begin{array}{c}
\text { (1) } \frac{\partial C_{T}}{\partial t}=k W C_{L}-p C_{T} \\
\text { (2) } \frac{\partial\left(C_{T}+\delta C_{T}\right)}{\partial t}+p\left(C_{T}+\delta C_{T}\right)=k W\left(C_{L}+\delta C_{L}\right) \\
\text { (3) } C_{T} \text { and } C_{L} \text { are known at } t \\
\text { (4) } \delta C_{T}(t)=\delta C_{L}(t)=0
\end{array}\right.
$$

This system leads to

$$
\left\{\begin{array}{c}
\text { for any } \Delta t, \frac{\partial\left(\delta C_{T}\right)}{\partial t}+p\left(\delta C_{T}\right)=k W\left(\delta C_{L}\right) \\
\delta C_{T}(t)=\delta C_{L}(t)=0
\end{array}\right.
$$

$\delta C_{T}$ is thus independent of both $C_{T}$ and $C_{L}$, and depends only on the $\Delta t$ value.

\section{Error induced by the GOA use}

In this section, the consequences of using the GOA on the computed solution accuracy are investigated in term of accuracy of the computed solution. In the following, $\theta_{T}$ refers to the exact solution while $\theta_{T}^{G O A}$ refers to the approximation of $\theta_{T}$ computed using the GOA.

\subsection{Error definition}

The error induced by the GOA is written as 


$$
E(t)=\frac{\theta_{T}^{G O A}(t)-\theta_{T}(t)}{\theta_{T}^{G O A}(t)}
$$

with $E \backslash l e f t(0 \backslash$ right $)=0$ (because the same initial conditions are considered for $\theta_{T}^{G O A}$ and $\left.\theta_{T}(t)\right)$.

The error $E$ can then be rewritten as (the definition of $\theta_{T}^{t r}, \theta_{T}^{t r, G O A}, \theta_{T}^{s t}$ and $\theta_{T}^{s t, G O A}$, respectively the transient and steady-state part of $\theta_{T}$ and $\theta_{T}^{G O A}$, are given in section 3.2 and 3.3)

$$
E(t)=\frac{\theta_{T}^{t r, G O A}(t)}{\theta_{T}^{G O A}(t)}\left(1-\frac{\theta_{T}^{t r}(t)}{\theta_{T}^{t r, G O A}(t)}\right)+\frac{\theta_{T}^{s t, G O A}(t)}{\theta_{T}^{G O A}(t)}\left(1-\frac{\theta_{T}^{s t}(t)}{\theta_{T}^{s t, G O A}(t)}\right)
$$

i.e., at $t=t_{n+1}$

$$
\begin{aligned}
E\left(t_{n+1}\right)= & \frac{\theta_{T}^{t r, G O A}\left(t_{n+1}\right)}{\theta_{T}^{G O A}\left(t_{n+1}\right)}\left(1-\frac{\theta_{T}^{t r}\left(t_{n+1}\right)}{\theta_{T}^{t r, G O A}\left(t_{n+1}\right)}\right) \\
& +\frac{\theta_{T}^{s t, G O A}\left(t_{n+1}\right)}{\theta_{T}^{G O A}\left(t_{n+1}\right)}\left(1-\frac{\theta_{T}^{s t}\left(t_{n+1}\right)}{\theta_{T}^{s t, G O A}\left(t_{n+1}\right)}\right)
\end{aligned}
$$

The term $1-\frac{\theta_{T}^{t r}\left(t_{n+1}\right)}{\theta_{T}^{t r, G O A}\left(t_{n+1}\right)}$ represents the error made with the GOA on the transient part of $\theta_{T}$, while $1-\frac{\theta_{T}^{s t}\left(t_{n+1}\right)}{\theta_{T}^{s t, G O A}\left(t_{n+1}\right)}$ denotes the error on the steady-state, i.e., the relevance of the Oriani's assumption $^{2}$ (the GOA being only an extension of this latter to include transient effects). Last, it is introduced $e(t) \in[0,1]$ so that $e\left(t_{n+1}\right)=\frac{\theta_{T}^{t r, G O A}\left(t_{n+1}\right)}{\theta_{T}^{G O A}\left(t_{n+1}\right)}$ and $1-e\left(t_{n+1}\right)=\frac{\theta_{T}^{s t, G O A}\left(t_{n+1}\right)}{\theta_{T}^{G O A}\left(t_{n+1}\right)}$, representing the relative weigh of $\theta_{T}^{t r, G O A}$ and $\theta_{T}^{s t, G O A}$ in $\theta_{T}^{G O A}$.

To be able to give an estimation of $E$, a recursive formulation is proposed in the following.

\subsection{Error estimation in the case of the McNabb \& Foster equation}

The incremental form of the theoretical solution of the McNabb \& Foster equation is (see Annex A), if $t_{n+1}=t_{n}+\Delta t$

$$
\begin{aligned}
\theta_{T}\left(t_{n+1}\right)= & \theta_{T}\left(t_{n}\right) \exp \left[-\int_{t_{n}}^{t_{n+1}}\left(p+k \theta_{L}\right) d u\right] \\
& +\left[F\left(t_{n+1}\right)-F\left(t_{n}\right)\right] \exp \left[-\int_{t_{n}}^{t_{n+1}}\left(p+k \theta_{L}\right) d u\right]
\end{aligned}
$$

while the GOA's one leads to

\footnotetext{
${ }^{2}$ As stated in [26], "Atomistically [this] assumption breaks down if the time in which a change, $\delta c_{L}$, occurs by lattice diffusion in the normal population is not sufficiently long to accomplish the change in the trapped population required by the equilibrium equation [(2.7)]".
} 


$$
\begin{gathered}
\theta_{T}^{G O A}\left(t_{n+1}\right)=\left[\theta_{T}^{G O A}\left(t_{n}\right)-\frac{k \theta_{L}\left(t_{n+1}\right)}{p+k \theta_{L}\left(t_{n+1}\right)}\right] e^{-\left[p+k \theta_{L}\left(t_{n+1}\right)\right] \Delta t}+\frac{k \theta_{L}\left(t_{n+1}\right)}{p+k \theta_{L}\left(t_{n+1}\right)} \\
=\theta_{T}^{G O A}\left(t_{n}\right) e^{-\left[p+k \theta_{L}\left(t_{n+1}\right)\right] \Delta t}+\frac{k \theta_{L}\left(t_{n+1}\right)}{p+k \theta_{L}\left(t_{n+1}\right)}\left(1-e^{-\left[p+k \theta_{L}\left(t_{n+1}\right)\right] \Delta t}\right)
\end{gathered}
$$

Both equations (3.4) and (3.5) exhibit a transient part and a steady state one on $\left[t_{n}, t_{n+1}\right]$, considering that $\theta_{T}=\theta_{T}^{t r}+\theta_{T}^{s t}$, with

leading to

$$
\theta_{T}^{s t}=\frac{k \theta_{L}}{k \theta_{L}+p}
$$

$$
\left\{\begin{array}{c}
\theta_{T}^{t r}\left(t_{n+1}\right)=\theta_{T}\left(t_{n}\right) \exp \left[-\int_{t_{n}}^{t_{n+1}}\left(p+k \theta_{L}\right) d u\right] \\
\theta_{T}^{t r, G O A}\left(t_{n+1}\right)=\theta_{T}^{G O A}\left(t_{n}\right) e^{-\left[p+k \theta_{L}\left(t_{n+1}\right)\right] \Delta t}
\end{array}\right.
$$

and

$$
\left\{\begin{array}{c}
\theta_{T}^{s t}\left(t_{n+1}\right)=\left[F\left(t_{n+1}\right)-F\left(t_{n}\right)\right] \exp \left[-\int_{t_{n}}^{t_{n+1}}\left(p+k \theta_{L}\right) d u\right] \\
\theta_{T}^{s t, G O A}\left(t_{n+1}\right)=\frac{k \theta_{L}\left(t_{n+1}\right)}{p+k \theta_{L}\left(t_{n+1}\right)}\left(1-e^{-\left[p+k \theta_{L}\left(t_{n+1}\right)\right] \Delta t}\right)
\end{array}\right.
$$

It can be seen that $\forall t, \theta_{T}^{s t}=\theta_{T}^{s t, G O A}$ for a constant $\theta_{L}$.

From equation (3.7) one can write

$$
\begin{aligned}
& \frac{\theta_{T}^{t r}\left(t_{n+1}\right)}{\theta_{T}^{t r, G O A}\left(t_{n+1}\right)}=\frac{\theta_{T}\left(t_{n}\right) \exp \left[-\int_{t_{n}}^{t_{n+1}}\left(p+k \theta_{L}\right) d u\right]}{\theta_{T}^{G O A}\left(t_{n}\right) e^{-\left[p+k \theta_{L}\left(t_{n+1}\right)\right] \Delta t}} \\
& =\left(1-E\left(t_{n}\right)\right) \exp \left[-\int_{t_{n}}^{t_{n+1}}\left(p+k \theta_{L}\right) d u+\left[p+k \theta_{L}\left(t_{n+1}\right)\right] \Delta \mathrm{t}\right]
\end{aligned}
$$

From the Mean theorem value, there exists $d \epsilon\left[t_{n}, t_{n+1}\right]$ so that

$$
-\int_{t_{n}}^{t_{n+1}} \theta_{L} d u+\theta_{L}\left(t_{n+1}\right) \Delta \mathrm{t}=\theta^{\prime}{ }_{L}(d) \frac{\Delta \mathrm{t}^{2}}{2}
$$

and equation (3.9) yields

$$
1-\frac{\theta_{T}^{t r}\left(t_{n+1}\right)}{\theta_{T}^{t r, G O A}\left(t_{n+1}\right)}=1-\left(1-E\left(t_{n}\right)\right) \exp \left[k \theta_{L}^{\prime}(d) \frac{\Delta \mathrm{t}^{2}}{2}\right]
$$

leading to

$$
\lim _{\Delta t \rightarrow 0} 1-\frac{\theta_{T}^{t r}\left(t_{n+1}\right)}{\theta_{T}^{t r, G O A}\left(t_{n+1}\right)}=\mathrm{E}\left(t_{n}\right)
$$

Furthermore (see Annex B), 


$$
\begin{gathered}
{\left[F\left(t_{n+1}\right)-F\left(t_{n}\right)\right] \exp \left[-\int_{t_{n}}^{t_{n+1}}\left(p+k \theta_{L}\right) d u\right]} \\
=\frac{k \theta_{L}\left(t_{n+1}\right)}{p+k \theta_{L}(c)}\left(1-e^{-\left[p+k \theta_{L}(c)\right] \Delta t}\right) \\
-k \theta^{\prime}{ }_{L}(d)\left\lfloor\frac{1}{\left(p+k \theta_{L}(c)\right)^{2}}\left(1-\mathrm{e}^{-\left(p+k \theta_{L}(c)\right) \Delta t}\right)-\frac{\Delta t \mathrm{e}^{-\left(p+k \theta_{L}(c)\right) \Delta t}}{\left(p+k \theta_{L}(c)\right)}\right\rfloor
\end{gathered}
$$

with $(c, d) \in\left[t_{n}, t_{n+1}\right]^{2}$, and

$$
\left.\begin{array}{c}
1-\frac{\theta_{T}^{s t}\left(t_{n+1}\right)}{\theta_{T}^{s t, G O A}\left(t_{n+1}\right)} \\
=1-\frac{p+k \theta_{L}\left(t_{n+1}\right)}{p+k \theta_{L}(c)} \times \frac{1-e^{-\left[p+k \theta_{L}(c)\right] \Delta t}}{1-e^{-\left[p+k \theta_{L}\left(t_{n+1}\right)\right] \Delta t}} \\
+\frac{\theta^{\prime}{ }_{L}(d)}{\theta_{L}\left(t_{n+1}\right)} \times \frac{p+k \theta_{L}\left(t_{n+1}\right)}{1-e^{-\left[p+k \theta_{L}\left(t_{n+1}\right)\right] \Delta t}} \\
\times\left\lfloor\frac{1}{\left(p+k \theta_{L}(c)\right)^{2}}\left(1-\mathrm{e}^{-\left(p+k \theta_{L}(c)\right) \Delta t}\right)-\frac{\Delta t \mathrm{e}^{-\left(p+k \theta_{L}(c)\right) \Delta t}}{\left(p+k \theta_{L}(c)\right)}\right.
\end{array}\right]
$$

It is worth underlining that (see equation (B.8))

$$
\lim _{\Delta \mathrm{t} \rightarrow 0} 1-\frac{\theta_{T}^{s t}\left(t_{n+1}\right)}{\theta_{T}^{s t, G O A}\left(t_{n+1}\right)}=0
$$

Thus, an upper bound value for $|E|$ can be found

$$
\begin{aligned}
& \leq \max _{(c, d) \epsilon\left[t_{n}, t_{n+1}\right]^{2}} \mid e\left(t_{n+1}\right)\left\{1-\left(1-E\left(t_{n}\right)\right) \exp \left[k \theta^{\prime}{ }_{L}(d) \frac{\left.\Delta \mathrm{t}^{2}\right]}{2}\right]\right\} \\
& +\left(1-e\left(t_{n+1}\right)\right)\left\{1-\frac{p+k \theta_{L}\left(t_{n+1}\right)}{p+k \theta_{L}(c)} \times \frac{1-e^{-\left[p+k \theta_{L}(c)\right] \Delta t}}{1-e^{-\left[p+k \theta_{L}\left(t_{n+1}\right)\right] \Delta t}}\right. \\
& \quad+\frac{\theta^{\prime}{ }_{L}(d)}{\theta_{L}\left(t_{n+1}\right)} \times \frac{p+k \theta_{L}\left(t_{n+1}\right)}{1-e^{-\left[p+k \theta_{L}\left(t_{n+1}\right)\right] \Delta t}} \\
& \left.\left.\left.\times \mid \frac{1}{\left(p+k \theta_{L}(c)\right)^{2}}\left(1-\mathrm{e}^{-\left(p+k \theta_{L}(c)\right) \Delta t}\right)-\frac{\Delta t \mathrm{e}^{-\left(p+k \theta_{L}(c)\right) \Delta t}}{\left(p+k \theta_{L}(c)\right)}\right]\right)\right\}
\end{aligned}
$$

This expression is rather complex, but depends only on known functions. If $\left|k \theta_{L}\right| \ll p$, equation (3.16) becomes

$$
\begin{gathered}
\leq \max _{(c, d) \epsilon\left[t_{n}, t_{n+1}\right]^{2}} \mid e\left(t_{n+1}\right)\left\{1-\left(1-E\left(t_{n}\right)\right) \exp \left[k \theta^{\prime}{ }_{L}(d) \frac{\Delta \mathrm{t}^{2}}{2}\right]\right\} \\
+\left(1-e\left(t_{n+1}\right)\right) \frac{\theta_{L}^{\prime}(d)}{\theta_{L}\left(t_{n+1}\right)} \times \frac{p}{1-e^{-p \Delta t}} \times\left[\frac{1}{p^{2}}\left(1-\mathrm{e}^{-p \Delta t}\right)-\frac{\Delta t \mathrm{e}^{-p \Delta t}}{p}\right] \mid
\end{gathered}
$$

while when $\left|k \theta_{L}\right| \gg p$, it becomes 


$$
\begin{gathered}
\leq \max _{(c, d) \epsilon\left[t_{n}, t_{n+1}\right]^{2}} \mid e\left(t_{n+1}\right)\left\{1-\left(1-E\left(t_{n}\right)\right) \exp \left[k \theta^{\prime}{ }_{L}(d) \frac{\Delta \mathrm{t}^{2}}{2}\right]\right\} \\
+\left(1-e\left(t_{n+1}\right)\right)\left\{1-\frac{\theta_{L}\left(t_{n+1}\right)}{\theta_{L}(c)} \times \frac{1-e^{-k \theta_{L}(c) \Delta t}}{1-e^{-k \theta_{L}\left(t_{n+1}\right) \Delta t}}\right. \\
\left.+\frac{k \theta_{L}^{\prime}(d)}{1-e^{-k \theta_{L}\left(t_{n+1}\right) \Delta t}} \times\left\lfloor\frac{1}{\left(k \theta_{L}(c)\right)^{2}}\left(1-\mathrm{e}^{-k \theta_{L}(c) \Delta t}\right)-\frac{\Delta t \mathrm{e}^{-k \theta_{L}(c) \Delta t}}{k \theta_{L}(c)}\right]\right\} \mid
\end{gathered}
$$

From equations (3.12) and (3.15), it can be concluded that $\forall \theta, E\left(t_{n+1}\right) \rightarrow e\left(t_{n+1}\right) E\left(t_{n}\right)$ when $\Delta t \rightarrow 0$, i.e.,

$$
E(t) \rightarrow\left(\prod_{k=1}^{t / \Delta t} e(k \Delta t)\right) E(0)
$$

As $E(0)=0$ and $0 \leq e(k \Delta t) \leq 1$, thus $\forall t, \lim _{\Delta t \rightarrow 0} E(t)=0$ : the GOA approaches the exact solution.

\subsection{Error estimation in the case of the Carter \& Kibler equation}

In the case of Carter \& Kibler trapping differential equation, the theoretical $\theta_{T}$ function is so that

$$
\theta_{T}\left(t_{n+1}\right)=\theta_{T}\left(t_{n}\right) \exp \mathrm{e}^{-p \Delta t}+\left[F\left(t_{n+1}\right)-F\left(t_{n}\right)\right] \mathrm{e}^{-p \Delta t}
$$

while the GOA's one leads to

$$
\begin{gathered}
\theta_{T}^{G O A}\left(t_{n+1}\right)=\left[\theta_{T}^{G O A}\left(t_{n}\right)-\frac{k}{p} \theta_{L}\left(t_{n+1}\right)\right] e^{-p \Delta t}+\frac{k}{p} \theta_{L}\left(t_{n+1}\right) \\
=\theta_{T}^{G O A}\left(t_{n}\right) e^{-p \Delta t}+\frac{k}{p} \theta_{L}\left(t_{n+1}\right)\left(1-e^{-p \Delta t}\right)
\end{gathered}
$$

The systems (3.7) and (3.8) become

and

$$
\left\{\begin{array}{c}
\theta_{T}^{t r}\left(t_{n+1}\right)=\theta_{T}\left(t_{n}\right) e^{-p \Delta t} \\
\theta_{T}^{t r, G O A}\left(t_{n+1}\right)=\theta_{T}^{G O A}\left(t_{n}\right) e^{-p \Delta t}
\end{array}\right.
$$

From equation (3.22),

$$
\left\{\begin{array}{c}
\theta_{T}^{s t}\left(t_{n+1}\right)=\left(\int_{t_{n}}^{t_{n+1}} k \theta_{L} \mathrm{e}^{p\left(u-t_{n}\right)} d u\right) e^{-p \Delta t} \\
\theta_{T}^{s t, G O A}\left(t_{n+1}\right)=\frac{k}{p} \theta_{L}\left(t_{n+1}\right)\left(1-e^{-p \Delta t}\right)
\end{array}\right.
$$




$$
1-\frac{\theta_{T}^{t r}\left(t_{n+1}\right)}{\theta_{T}^{t r, G O A}\left(t_{n+1}\right)}=1-\frac{\theta_{T}\left(t_{n}\right)}{\theta_{T}^{G O A}\left(t_{n}\right)}=E\left(t_{n}\right)
$$

while

$$
\frac{\theta_{T}^{s t}\left(t_{n+1}\right)}{\theta_{T}^{s t, G O A}\left(t_{n+1}\right)}=\frac{\left(\int_{t_{n}}^{t_{n+1}} k \theta_{L} \mathrm{e}^{p\left(u-t_{n}\right)} d u\right) e^{-p \Delta t}}{\frac{k}{p} \theta_{L}\left(t_{n+1}\right)\left(1-e^{-p \Delta t}\right)}
$$

which can be rewritten as (see annex C)

$$
1-\frac{\theta_{T}^{s t}\left(t_{n+1}\right)}{\theta_{T}^{s t, G O A}\left(t_{n+1}\right)}=\frac{k \theta^{\prime}(c)\left[\frac{1}{p^{2}}\left(1-\mathrm{e}^{-p \Delta t}\right)-\frac{\Delta t}{p} \mathrm{e}^{-p \Delta t}\right]}{\frac{k}{p} \theta_{L}\left(t_{n+1}\right)\left(1-e^{-p \Delta t}\right)}
$$

with $c \in\left[t_{n}, t_{n+1}\right]$. It can be observed that:

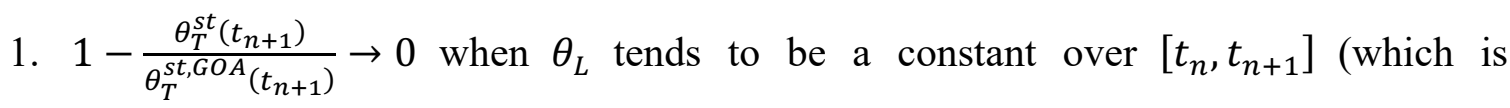
consistent with the Oriani's assumption domain of validity);

2. $1-\frac{\theta_{T}^{s t}\left(t_{n+1}\right)}{\theta_{T}^{s t, G O A}\left(t_{n+1}\right)} \rightarrow 0$ when $\Delta t \rightarrow 0$ (see equation (C.6));

3. $1-\frac{\theta_{T}^{S t}\left(t_{n+1}\right)}{\theta_{T}^{s t, G O A}\left(t_{n+1}\right)}$ does not depend on $k$.

Equation (3.3) is consequently equivalent to

$$
E\left(t_{n+1}\right)=e\left(t_{n+1}\right) E\left(t_{n}\right)+\left(1-e\left(t_{n+1}\right)\right) \frac{\theta^{\prime}{ }_{L}(c)\left[\frac{1}{p}\left(1-\mathrm{e}^{-p \Delta t}\right)-\Delta t \mathrm{e}^{-p \Delta t}\right]}{\theta_{L}\left(t_{n+1}\right)\left(1-e^{-p \Delta t}\right)}
$$

and an upper bound value for $|E|$ can be found

$$
\leq \max _{c \in\left[t_{n}, t_{n+1}\right]}\left|e\left(t_{n+1}\right) E\left(t_{n}\right)+\left(1-e\left(t_{n+1}\right)\right) \frac{\theta^{\prime}{ }_{L}(c)\left[\frac{1}{p}\left(1-\mathrm{e}^{-p \Delta t}\right)-\Delta t \mathrm{e}^{-p \Delta t}\right]}{\theta_{L}\left(t_{n+1}\right)\left(1-e^{-p \Delta t}\right)}\right|
$$

which corresponds to equation (3.17). From the point 2, it can be concluded that $E\left(t_{n+1}\right)$ $\rightarrow e\left(t_{n+1}\right) E\left(t_{n}\right)$ when $\Delta t \rightarrow 0$, i.e.,

$$
\mathrm{E}(\mathrm{t}) \rightarrow\left(\prod_{k=1}^{t / \Delta t} e(k \Delta t)\right) E(0)
$$

As $E(0)=0$ and $0 \leq e(k \Delta t) \leq 1$, thus $\forall t, \lim _{\Delta t \rightarrow 0} E(t)=0$ : the GOA tends toward the exact solution.

$E(t)$ bounds are controlled by, for $p \Delta t \ll 1$, the function $\Delta t^{2} \theta^{\prime}{ }_{L}$, and especially its maximum and minimum values other each interval $\left[t_{n}, t_{n+1}\right]$ (see Annex C). 


\section{Application}

To illustrate the relationship between the time increment, the $\theta_{L}$ function and the GOA's error, an illustration is proposed in this section. Only the trapping equation is considered: $\theta_{L}$ is not the result of a coupled transport and trapping problem, but imposed and set a priori (even if theoretical results can be found for both $\theta_{L}$ and $\theta_{T}$ for specific configurations [24]). $\theta_{L}$ is set as a sinus function

$$
\theta_{L}=\mathrm{A} \frac{1-\sin \omega t}{2}
$$

This relationship for $\theta_{L}$ insure that, $\forall t, \theta_{L}>0$, to be consistent with the physical variation domain for $C_{L}$. Its maximum value is set to $A$, leading to an initial $\theta_{L}$ value equal to $A / 2$. For the sake of simplicity in the parametric study, only this arbitrary condition is considered in the following.

It is worth noting that equation (2.6)b1 can be rewritten in a dimensionless matter: if $A$ is set as the maximum value of $\theta_{L}(>0)$ and $1 / \omega$ its critical time, thus equation (2.6)b1 becomes

$$
\frac{\partial \theta_{T}}{\partial \tau}=\kappa \tilde{\theta}_{L}(\tau)\left(1-\theta_{T}\right)-\beta \theta_{T}
$$

where $\tilde{\theta}_{L}=\theta_{L} / A$, and $\tau=\omega t$ is a dimensionless time, i.e.

$$
\tilde{\theta}_{L}=\frac{1-\sin \tau}{2}
$$

with a characteristic time equal to $\pi . \kappa$ is equal to $k A \omega$ and $\beta=p \omega$.

In the following, for the sake of illustration, $(\kappa, \beta)$ is so that $(\kappa, \beta) \in\left\{10^{-3}, 10^{3}\right\}^{2}$, and the imposed time increment $\Delta \tau$ so that $\Delta \tau \in\left\{\pi, \frac{\pi}{5}, \frac{\pi}{50}, \frac{\pi}{500}\right\}$. It is worth noting that, as $\beta$ (or $p$ ) is linked to the trapping energy (see [1]), performing a parametric study on $\beta$ is equivalent to considering various trap kind (reversible or not).

For each configuration $(\kappa, \beta, \Delta \tau)$, the theoretical $\theta_{T}$ solution is computed, based on Python scripts (and the odeint library [51]), as well as the GOA's one. Then, the exact error $|E|$ as well as the estimated upper bounds (equation (3.18) and (3.28) for the McNabb \& Foster and the Carter \& Kibler equations, respectively) have been plotted.

\subsection{McNabb \& Foster equation}

On Figure 2 are plotted the results for $\theta_{T}(\tau)$, and on Figure 3 the one for $|E(\tau)|$. On Figure 2 are also plotted, in grey, the $\tilde{\theta}_{L}$ evolution with $\tau$.

For all the $(\kappa, \beta)$ values, it can first be seen that the exact $\theta_{T}$ evolution is well approached by the GOA, for all the considered $\Delta \tau$. For $\beta=10^{3}$ (Figure $2 \mathrm{~b}$ and $\mathrm{c}$ ), whatever $\kappa$, the Oriani's solution is found, denoting slow $\tilde{\theta}_{L}$ temporal variations compared to the trapping process kinetic. Low $\beta$ values correspond to different configurations: when $\kappa$ is low (Figure 2a), the trapping process is a transient one, $\theta_{T}$ increasing slowly toward a steady state that can be close to the one plotted on Figure $2 \mathrm{~d}$. This last configuration exhibits a -almost- constant $\theta_{T}$, insensitive to $\tilde{\theta}_{L}$ temporal variations: the trapping kinetic is too slow to reflect these variations.

While focusing on the $|E(\tau)|$ values, it can be observed that a decreasing $\Delta \tau$ leads to a decreasing computed $|E(\tau)|$. As expected, the $\theta_{T}$ estimation by the GOA tends toward the exact solution when $\Delta \tau$ tends toward 0 . It can be observed that the proposed upper bound 
(equation (3.18)) tends to give a correct $|E(\tau)|$ estimation for the smaller $\Delta \tau$ values (i.e., for errors bellow 0.01$)$. For high $\kappa$ values, the $|E(\tau)|$ upper bound tends to overestimates the error, regardless of $\Delta \tau$, even if the GOA allows a very good evaluation of $\theta_{T}$. It must however be underlined that the proposed upper bound allows to control the effective error for all the $(\kappa, \beta)$ values.

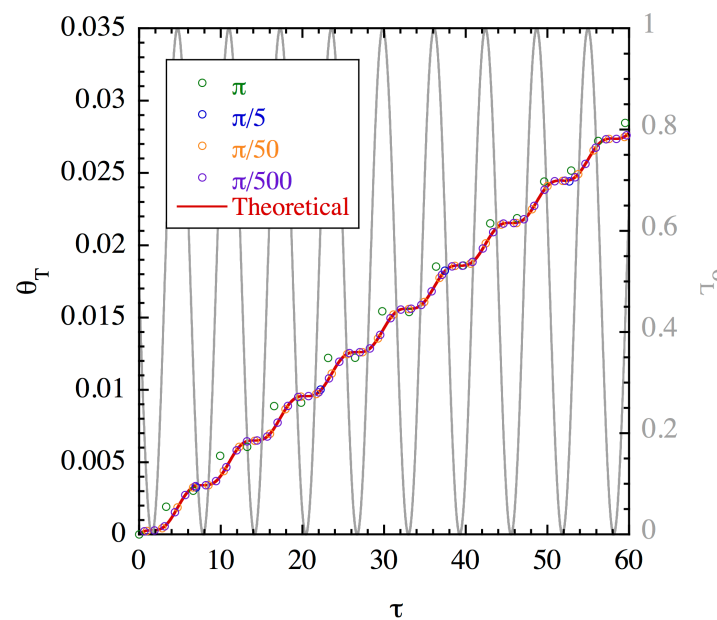

(a) $\kappa=\beta=10^{-3}$

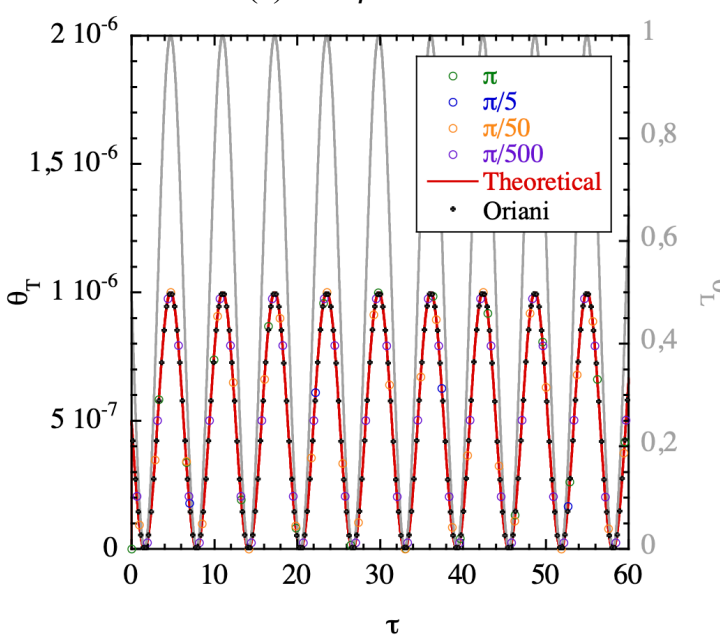

(c) $\kappa=10^{-3}$ and $\beta=10^{3}$

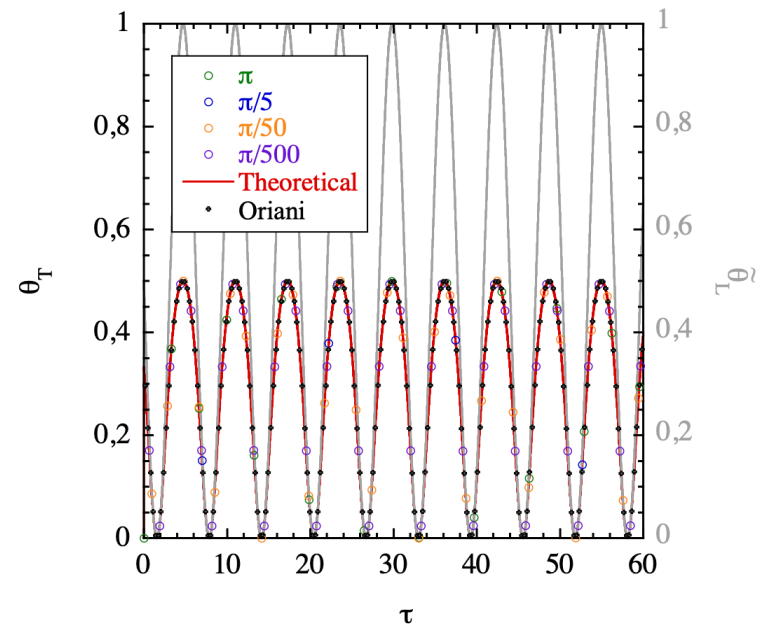

(b) $\kappa=\beta=10^{3}$

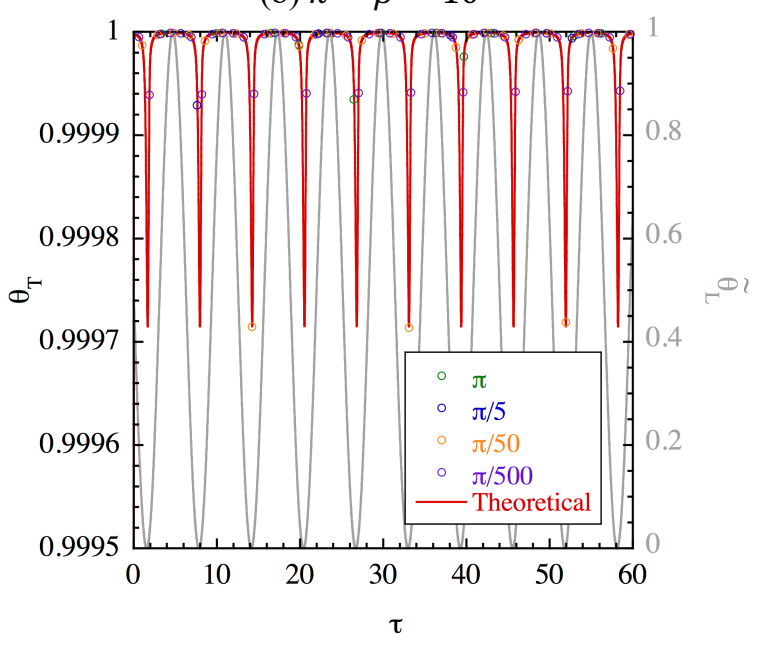

(d) $=10^{3}$ and $\beta=10^{-3}$

Figure 2. Influence of $\Delta \tau$ on the evaluation of $\theta_{T}$ by the GOA for several $\kappa$ and $\beta$ values. For the sake of comparison, the Oriani's solution has also been plotted for (b) and (c) (black dots). 

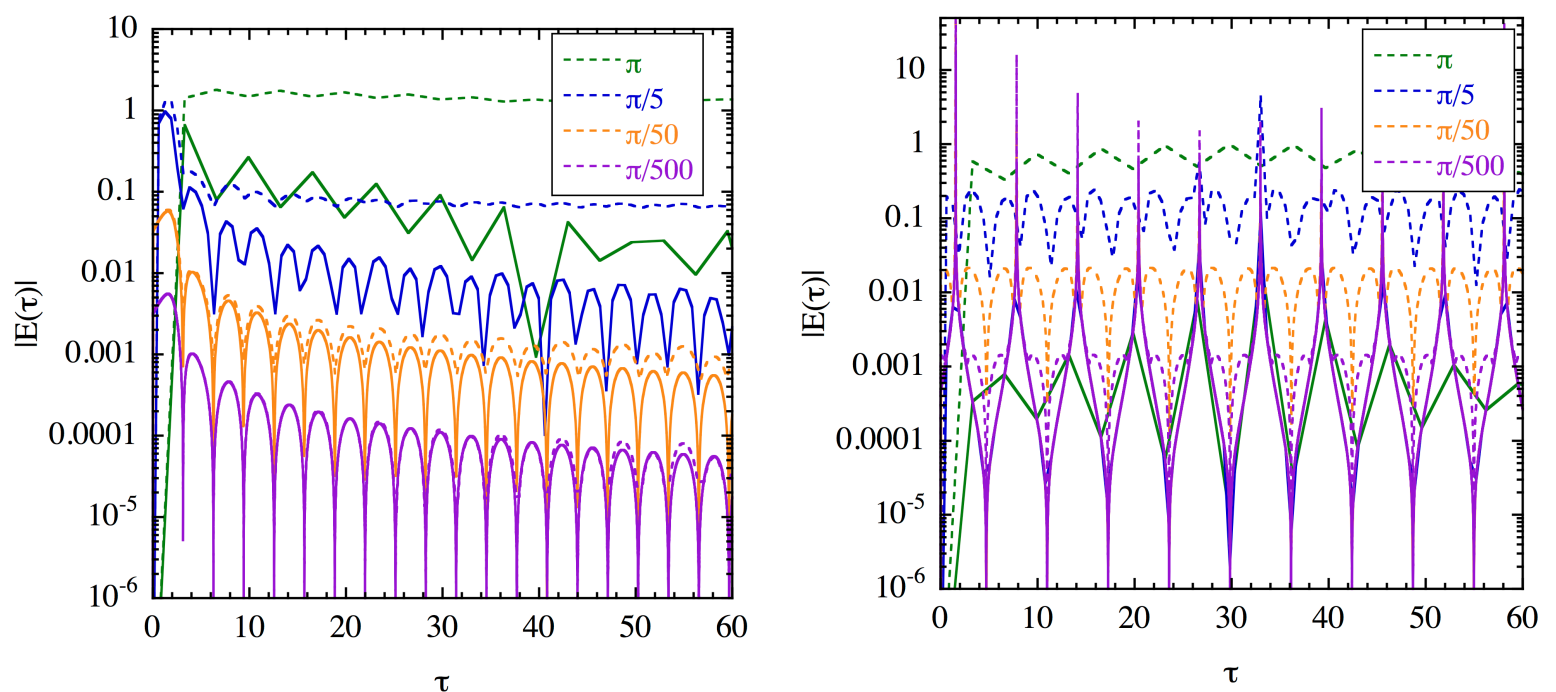

(a) $\kappa=\beta=\stackrel{\tau}{10} 0^{-3}$

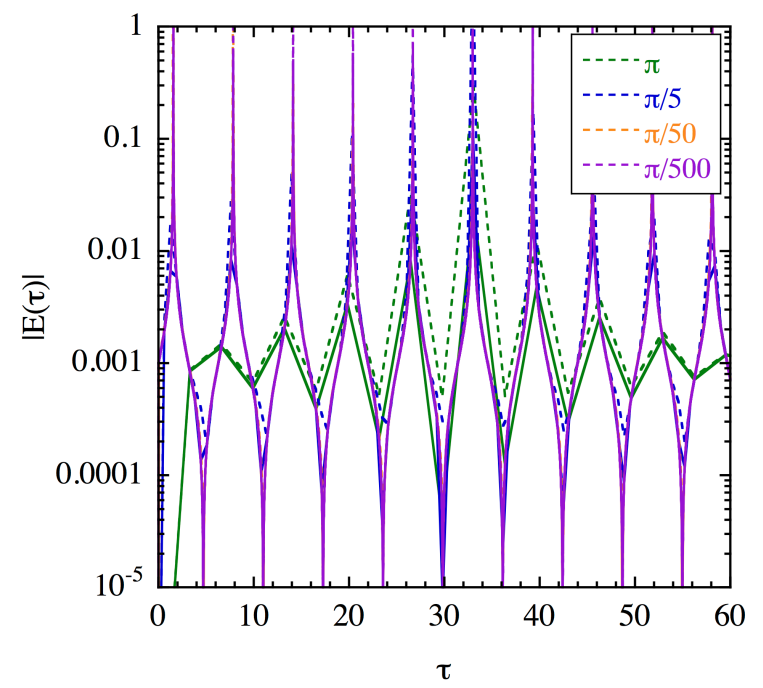

(c) $\kappa=10^{-3}$ and $\beta=10^{3}$

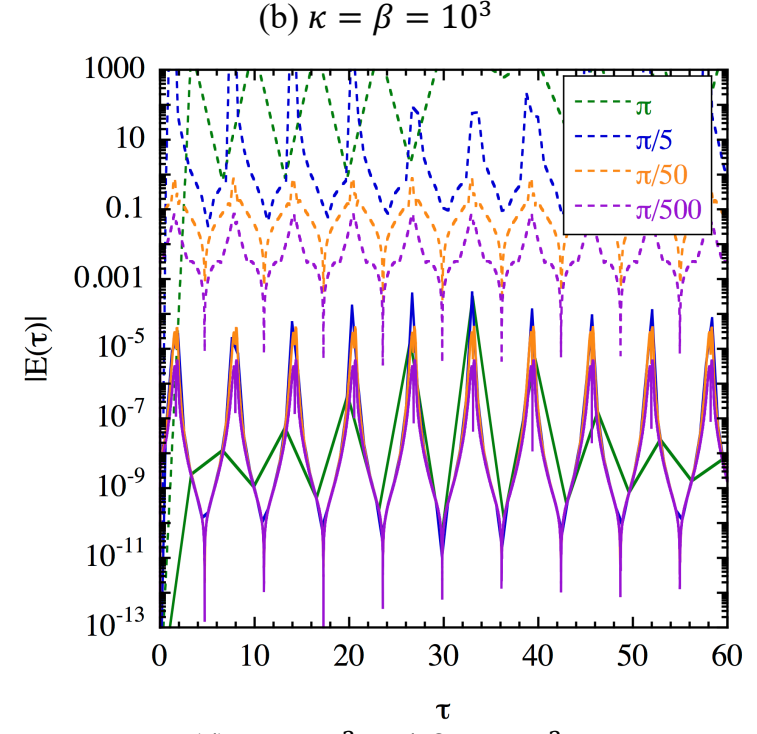

(d) $\kappa=10^{3}$ and $\beta=10^{-3}$

Figure 3. Influence of $\Delta \tau$ on the evaluation of $E(\tau)$ (continuous line) and its upper bound (dashed line equation (3.18)) for several $\kappa$ and $\beta$ values.

\subsection{Carter \& Kibler equation}

The results for $\theta_{T}(\tau)$ and $|E(\tau)|$ are plotted on Figure 4 and Figure 5 respectively.

On Figure 4 are plotted the results for $\theta_{T}(\tau)$ and on Figure 5 the one for $|E(\tau)|$.

As in the previous section, the GOA allows to get a good estimation of the theoretical solution. Moreover, it can be observed that, as in the previous section, for $\beta=10^{3}$ (Figure $4 \mathrm{~b}$ and $\mathrm{c}$ ), the steady-state solution $\theta_{T}=\frac{\kappa}{\beta} \tilde{\theta}_{L}(\tau)$ is found, and that for $\kappa=10^{-3}$ (Figure 4a and c), no differences between the McNabb \& Foster and Carter \& Kibler results can be seen (because $\left.1-\theta_{T} \approx 1\right)$. The main differences with the previous section can be seen Figure $4 \mathrm{~d}$ : as the trapping is here not bounded, $\theta_{T}$ continuously increasing toward $+\infty$.

The $|E(\tau)|$ upper bound evaluation appears to be, as expected from equation (3.28), $\kappa$ independent. A decreasing $\Delta \tau$ leads to a convergence of this upper bound toward $|E(\tau)|$, allowing a control of the error. 


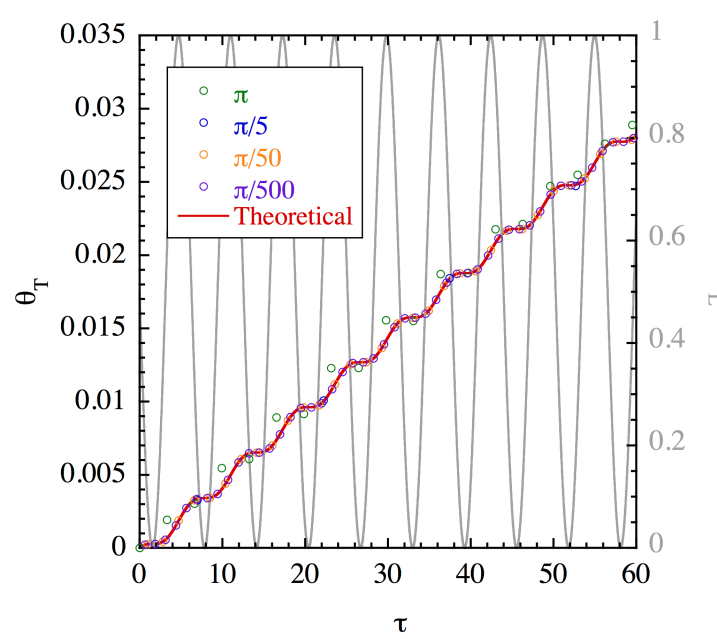

(a) $\kappa=\beta=10^{-3}$

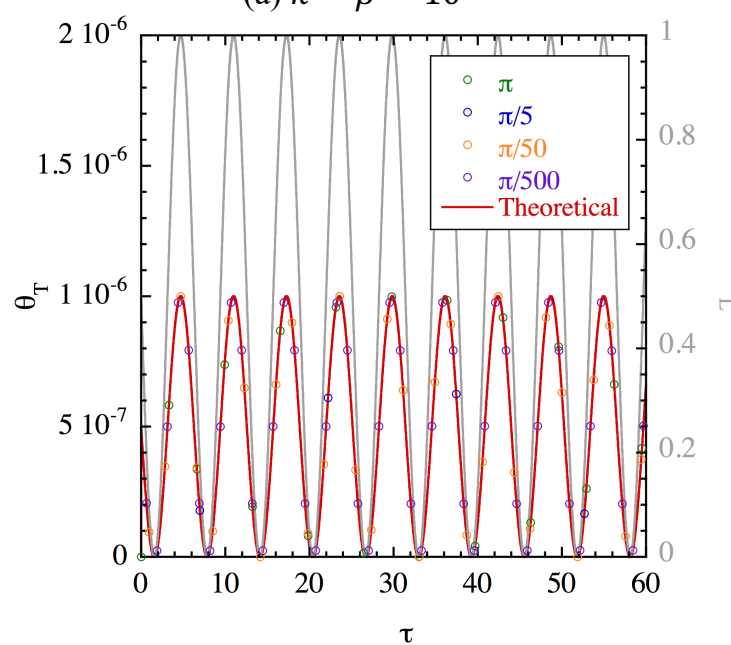

(c) $\kappa=10^{-3}$ and $\beta=10^{3}$

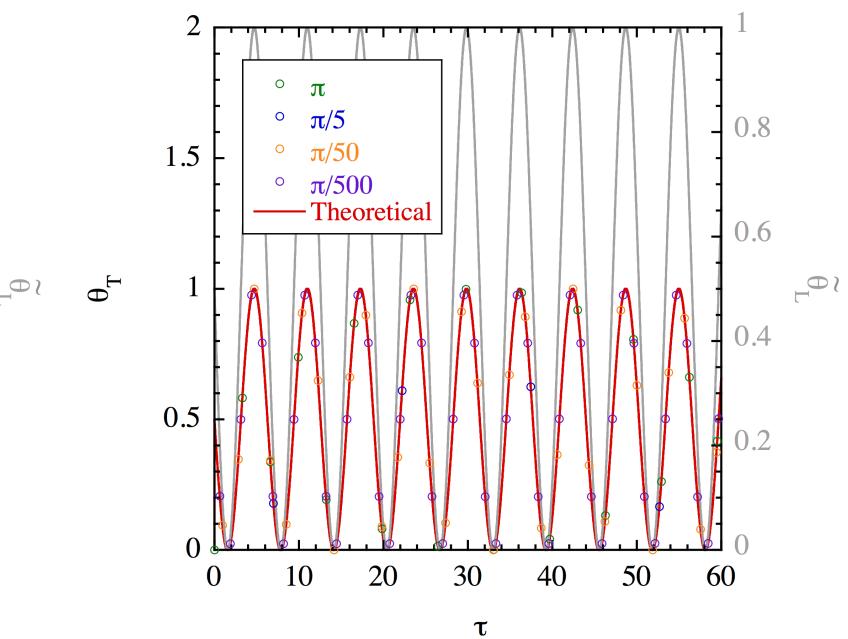

(b) $\kappa=\beta=10^{3}$

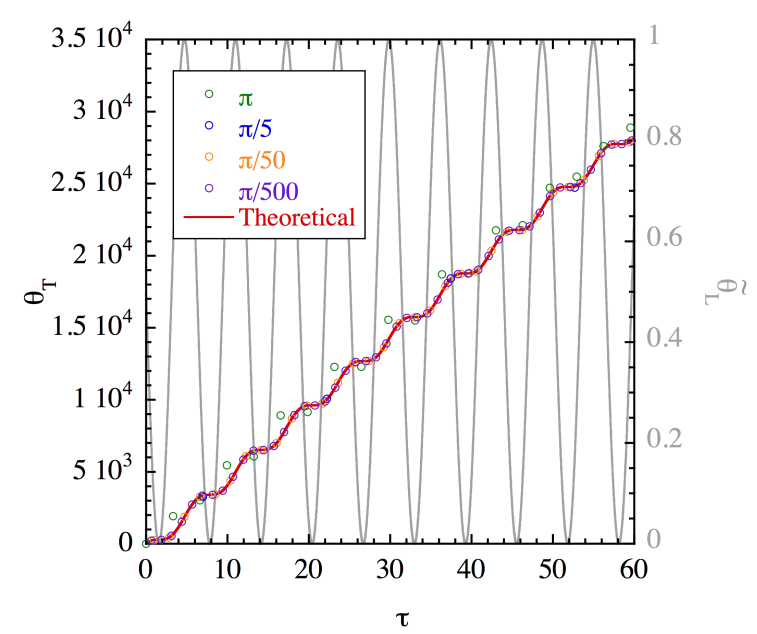

(d) $\kappa=10^{3}$ and $\beta=10^{-3}$

Figure 4. Influence of $\Delta \tau$ on the evaluation of $\theta_{T}$ by the GOA for several $\kappa$ and $\beta$ values. 


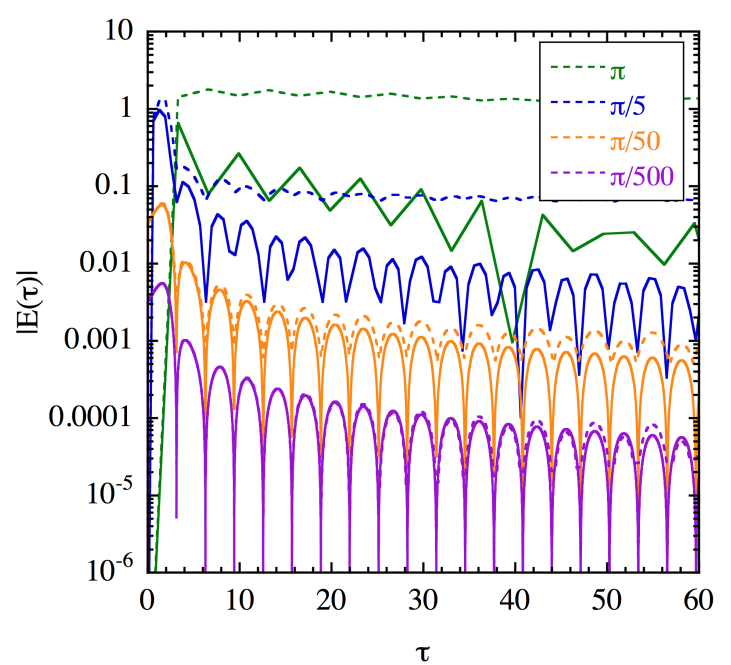

(a) $\kappa=\beta=10^{-3}$

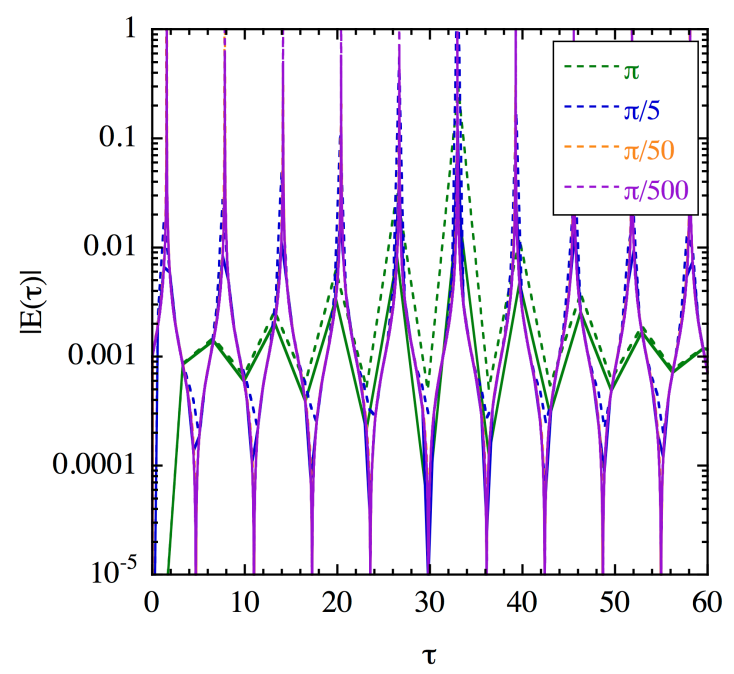

(c) $\kappa=10^{-3}$ and $\beta=10^{3}$

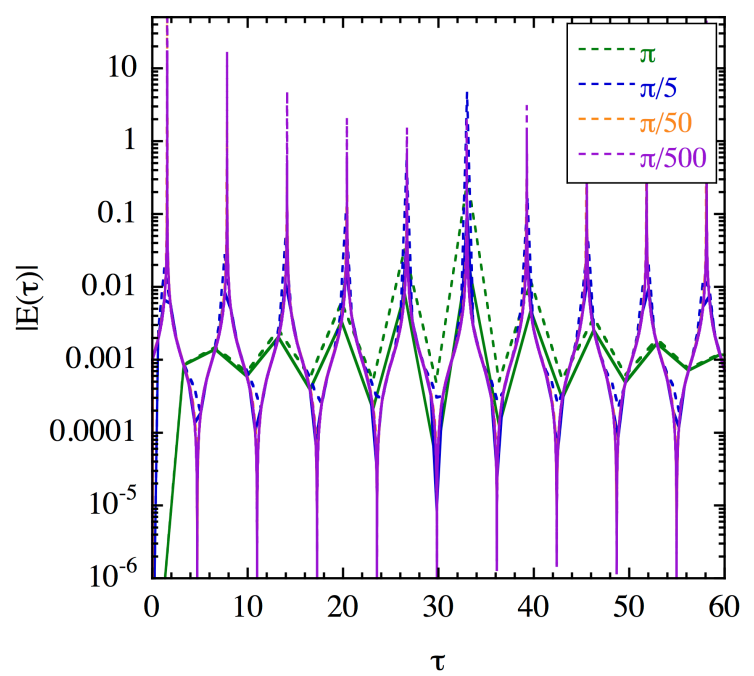

(b) $\kappa=\beta=10^{3}$

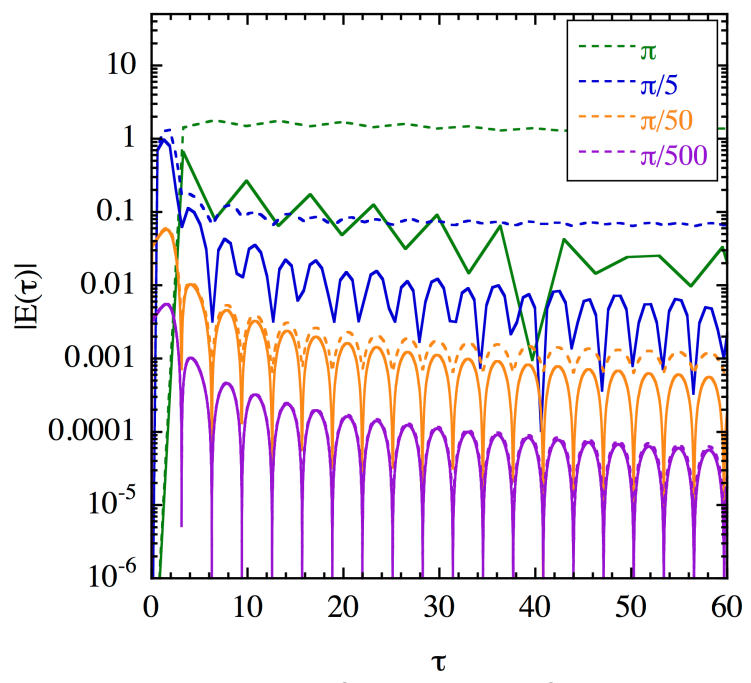

(d) $\kappa=10^{3}$ and $\beta=10^{-3}$

Figure 5. Influence of $\Delta \tau$ on the evaluation of $E(\tau)$ (continuous line) and its upper bound (dashed line equation (3.28)) for several $\kappa$ and $\beta$ values.

\subsection{Conclusion}

From the chosen configuration, it can be seen that the GOA allows a good approximation of the exact solution the differential equations describing the trapping kinetic of hydrogen in metals or water in polymers.

The proposed upper bound for the error generated when using the GOA appears to be very accurate for small time increments (with errors below 1\%), but for the McNabb \& Foster equation with important $\kappa$ and small $\beta$ values. Since $\kappa$ and $\beta$ corresponds to respectively the trapping and detrapping processes, thus, this upper bound estimation must be used cautiously in configurations where the difference between the trapping and detrapping energy is important. The estimated $\theta_{T}$ and the related error are however not affected by this limitation.

Last, it is worth noting that the proposed error upper bound is only dependent on known functions, and can consequently be used in configurations in which the $\tilde{\theta}_{L}$ variations are only known for the current time increment (e.g., when the diffusion process is also computed). 


\section{Abaqus implementation}

The main motivation to propose the GOA to solve the trapping differential equations equation (2.6)b was to provide Abaqus with an accurate $d u d t$ value during the computation. The importance of that parameter for the software convergence, when a UMATHT subroutine is used, is focused on this section. The transport and trapping system is derived from equation (2.6)

$$
\left\{\begin{array}{c}
(a) \frac{1}{W} \frac{\partial \theta_{L}}{\partial t}+\frac{\partial \theta_{T}}{\partial t}-D_{L} \frac{1}{W} \nabla \cdot\left(\nabla \theta_{L}\right)=0 \\
(b 1) \frac{\partial \theta_{T}}{\partial t}=k W \frac{\theta_{L}}{W}\left(1-\theta_{T}\right)-p \theta_{T} \\
\text { (b2) } \frac{\partial \theta_{T}}{\partial t}=W k \frac{\theta_{L}}{W}-p \theta_{T}
\end{array}\right.
$$

for which $c_{L}=\theta_{L} / W$ and $c_{T}=\theta_{T}$ spatio-temporal evolutions are sought. $W$ is, as previously, equal to $N_{T} / N_{L}$.

\subsection{Configuration}

For the sake of simplicity, the used configuration is described on Figure 6: it consists in a bar with a unity dimension, with a symmetry boundary condition at $x=0$ (i.e., zero normal hydrogen flux). At $t=0, \theta_{T}=0$ everywhere in the bar, as well as $\theta_{L}$ but for $x=1$, where $\theta_{L}=W$. For this configuration, an analytical solution exists for $c_{L}$ and $c_{T}$ in the case of water in polymers [24].

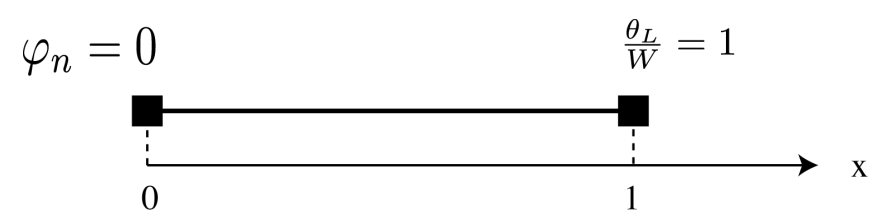

Figure 6. Geometric configuration and boundary conditions.

The parameters used in the computations are the one listed in Table $1,(p, k)$ parameters have been inspired by the hydrogen diffusion and trapping ones in tungsten [1]; this set of parameters does not intends to consistently reproduce hydrogen and trapping in tungsten system, and is only set for illustration.

Table 1. Trapping parameters.

\begin{tabular}{|c|c|c|c|}
\hline$D_{L}\left(\mathrm{~mm}^{2} \cdot \mathbf{s}^{-1}\right)$ & $k\left(\mathbf{s}^{-1}\right)$ & $p\left(\mathbf{s}^{-1}\right)$ & $W$ \\
\hline 1 & $8.01 \times 10^{5}$ & $3.6 \times 10^{-2}$ & $10^{-3}$ \\
\hline
\end{tabular}

This problem has been modeled in Abaqus 6.11 FE software, in 3D, the section of the bar being $0.01 \times 0.01 .8$ nodes full integration cubic tri-linear elements have been used, the dimensions of which being $0.005^{3}$. The Abaqus convergence criteria have been set as their standard values. Boundary conditions have been defined to reproduce the 1D problem given on Figure $6:$ a symmetry boundary condition at $x=0$ and $\theta_{L}=W$ at $x=1$. It is worth noting that $D_{L}=1 \mathrm{~mm}^{2} / \mathrm{s}$ and a unit-length bar are related to a dimensionless configuration.

The Finite Element implementation is described in section 2.2, using the GOA-based $d u d t$ estimation as provided by equations (2.14) for hydrogen in metals (McNabb \& Foster equation) and (2.21) for water in polymers (Carter \& Kibler equation). Because of the existence of an 
analytical solution, the Carter \& Kibler equation is first focused on and then the McNabb \& Foster's one.

\subsection{Carter \& Kibler trapping equation}

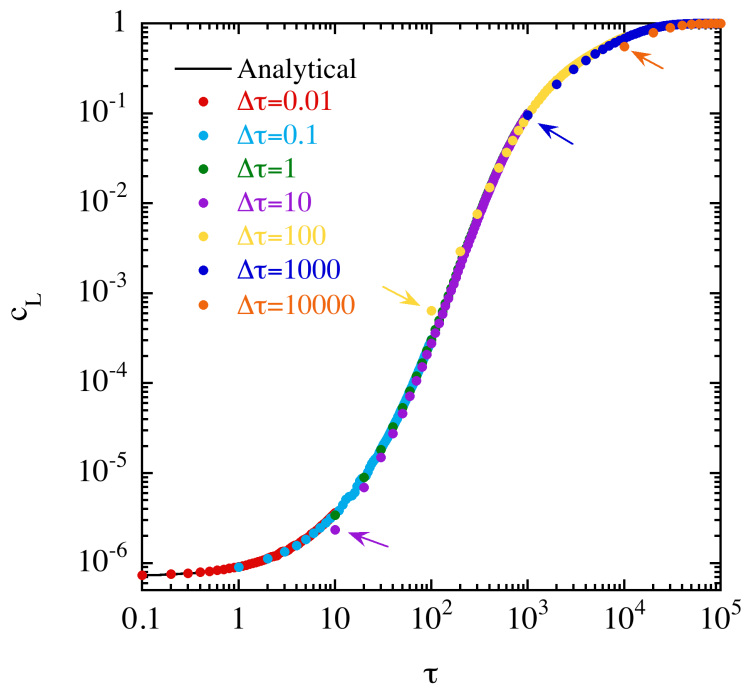

(a)

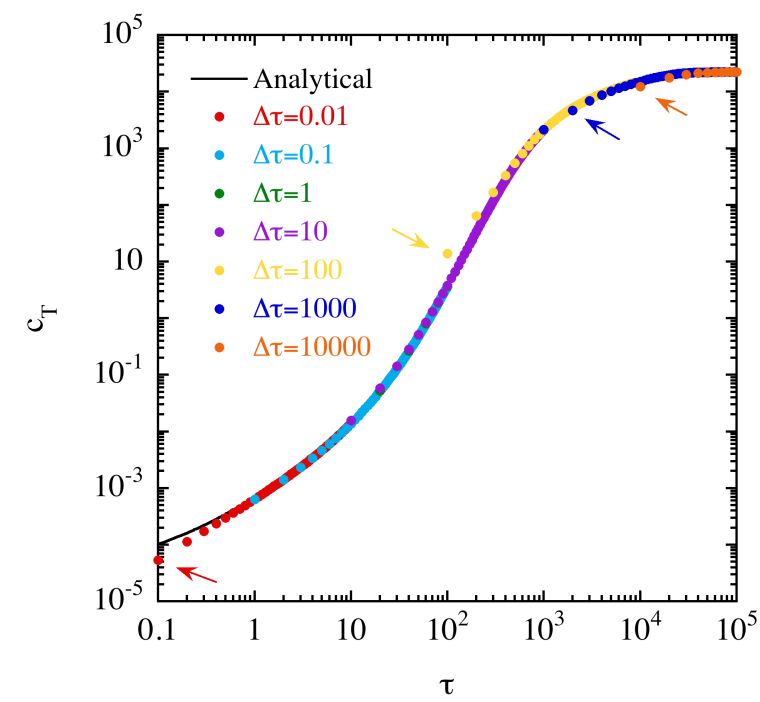

(b)

Figure 7. Comparison the theoritical and the FE-based variation with time of (a) $c_{L}$ and (b) $c_{T}$ at $x=0.5$. Several fixed times increment have been considered in the FE computation ( $\Delta t=0.01$ up to $10000 \mathrm{~s}$ ).

For the configuration presented on Figure $6, c_{T}$ and $c_{L}$ are so that [24]

$$
c_{T}=\frac{k W}{p}\left[1-\frac{4}{\pi} \sum_{i=1}^{\infty} \frac{(-1)^{\frac{i-1}{2}}}{i\left(r_{i}^{+}-r_{i}^{-}\right)}\left[r_{i}^{+} \exp \left(-r_{i}^{-} t\right)-r_{i}^{-} \exp \left(-r_{i}^{+} t\right)\right] \cos \left(\frac{\pi i}{2} x\right)\right]
$$

and

$$
\begin{aligned}
= & 1-\frac{4}{\pi} \sum_{i=1}^{\infty \text { (odd })} \frac{(-1)^{\frac{i-1}{2}}}{i\left(r_{i}^{+}-r_{i}^{-}\right)}\left[r_{i}^{+} \exp \left(-r_{i}^{-} t\right)-r_{i}^{-} \exp \left(-r_{i}^{+} t\right)\right] \cos \left(\frac{\pi i}{2} x\right) \\
& +\frac{4}{\pi \beta} \sum_{i=1}^{\infty(o d d)} \frac{(-1)^{\frac{i-1}{2}}}{i\left(r_{i}^{+}-r_{i}^{-}\right)} r_{i}^{+} r_{i}^{-}\left[\exp \left(-r_{i}^{-} t\right)-\exp \left(-r_{i}^{+} t\right)\right] \cos \left(\frac{\pi i}{2} x\right)
\end{aligned}
$$

$r_{i}^{-}$and $r_{i}^{-}$being equal to 


$$
\left\{\begin{array}{l}
r_{i}^{+}=\frac{1}{2}\left[\left(\frac{\pi^{2}}{4} i^{2}+k W+p\right)+\sqrt{\left(\frac{\pi^{2}}{4} i^{2}+k W+p\right)^{2}-\pi^{2} p i^{2}}\right] \\
r_{i}^{-}=\frac{1}{2}\left[\left(\frac{\pi^{2}}{4} i^{2}+k W+p\right)-\sqrt{\left(\frac{\pi^{2}}{4} i^{2}+k W+p\right)^{2}-\pi^{2} p i^{2}}\right.
\end{array}\right.
$$

\section{Results using the GOA's $d u d t$}

Theoretical and numerical $c_{T}$ and $c_{L}$ functions (estimated using the GOA) are plotted on Figure 7 , for various fixed time increments $\Delta t$, from $10^{-2}$ to $10^{4} \mathrm{~s}$.

For all fixed time increments, the FE problem has converged and it can be observed that the numerical results are able to reproduce the theoretical ones. It is worth noting that a slight difference can appear for the first increments of FE results (indicated by arrows on Figure 7). For all $\Delta t<10000 \mathrm{~s}$, at each increment, the FE problem has converged after one NewtonRaphson loop (except the second increment, which converge after two loops). For $\Delta t=10000$ $\mathrm{s}$, two loops are needed. On Figure 8 are plotted the comparison between the GOA and the numerical $\partial \mathrm{c}_{T} / \partial \mathrm{c}_{L}$ estimation for each $\Delta t$. This numerical approximation has been computed using

$$
\left.\frac{\partial c_{T}}{\partial c_{L}}\right|_{\text {num }}(\tau+\Delta \tau)=\frac{c_{T}\left(c_{L}+0.001, \tau+\Delta \tau\right)-c_{T}\left(c_{L}, \tau+\Delta \tau\right)}{0.001}
$$

where $c_{L}=c_{L}(\tau+\Delta \tau)(=$ temp + dtemp $)$.

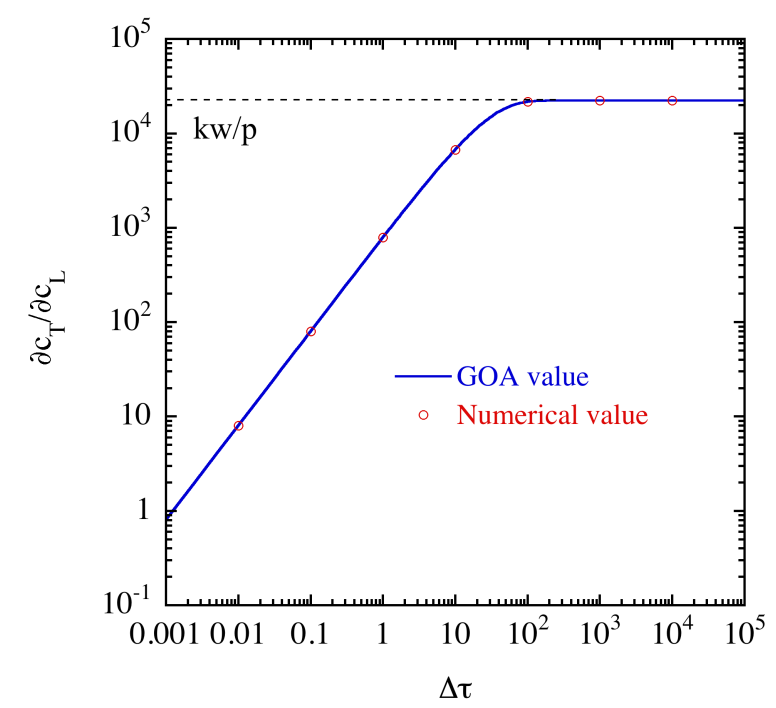

Figure 8. Comparison between the $\partial \mathrm{c}_{T} / \partial \mathrm{c}_{L}$ velue computed using the GOA formulation and equation (5.5).

It can be seen that numerical $\partial \mathrm{c}_{T} / \partial \mathrm{c}_{L}$ are constant as expected (see equation (2.21)), and equal to their theoretical values. Computing the correct value does not guarantee a perfect result (highlighted by arrows on Figure 7), which is linked to the accuracy of the GOA approximation, but only a fast convergence of the Newton-Raphson scheme (in one or two loops). 
2. Influence on $d u d t$ value on the convergence

The previous computations have been remade with two fixed $d u d t$ values to underline the influence of this parameter on FE results: 1 and $1+k W / p$. These values correspond respectively to the $d u d t$ value for $\Delta t \rightarrow 0^{+}$and $\Delta t \rightarrow+\infty$.

Table 2. Results of the FE computations using a constant $d u d t$. Convergence with a constant time increment are indicated in green (and non convergence in orange).

\begin{tabular}{c|l|l|l|l|l|l|l}
$\Delta u t(\mathrm{~s})$ & 0.01 & 0.1 & 1 & 10 & 100 & 1000 & 10000 \\
\hline 1 & & & & & & & \\
\hline $1+k W / p$ & & & & & & &
\end{tabular}

When using the previous fixed time increments, few computations have converged, as summarized on Table 2. The results of these computations are the same than the one on Figure 7.

For $d u d t=1+k W / p$ and $\Delta t \geq 100$, convergence has been able to be reached because, for $\Delta t \geq 100 \mathrm{~s}$,

$$
d u d t=1+\frac{k W}{p}\left(1-e^{-\beta \Delta \tau}\right) \approx 1+\frac{k W}{p}
$$

and thus, the constant $d u d t$ FE configuration is close to the theoretical $d u d t$ (equation (2.21)).

It is worth noting that, when the time increment is not fixed, convergence can be achieved in FE computations with $d u d t=1$, with results equivalent to the ones plotted on Figure 7 . The needed time increment for $d u d t=1$ is approximately equal to $\Delta t=10^{-3}-10^{-4}$ (i.e, small enough to get the effective $d u d t$ value close to 1 ), and it is required between 1 and 7 Newton-Raphson loops to get a solution at each increment. Any attempts of the software to increase the time increment leads to a cutback. As a consequence, the analytical evaluation of $d u d t$ provided by the GOA is not mandatory in the case of Carter \& Kibler equation, although it allows a much more efficient convergence.

No extra configuration than the one indicates in Table 2 leads to convergence for $d u d t=1+$ $k W / p$.

\subsection{McNabb \& Foster trapping equation}

Temporal evolution of $c_{T}$ and $c_{L}$ have been estimated at $x=0.5$, for several fixed time increment, and the results plotted on Figure 9 at $x=0.5$. 


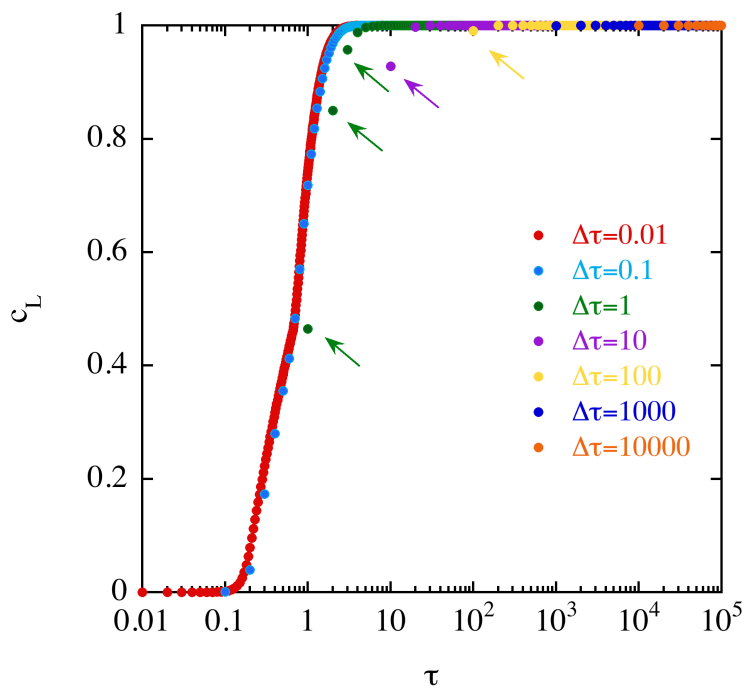

(a)

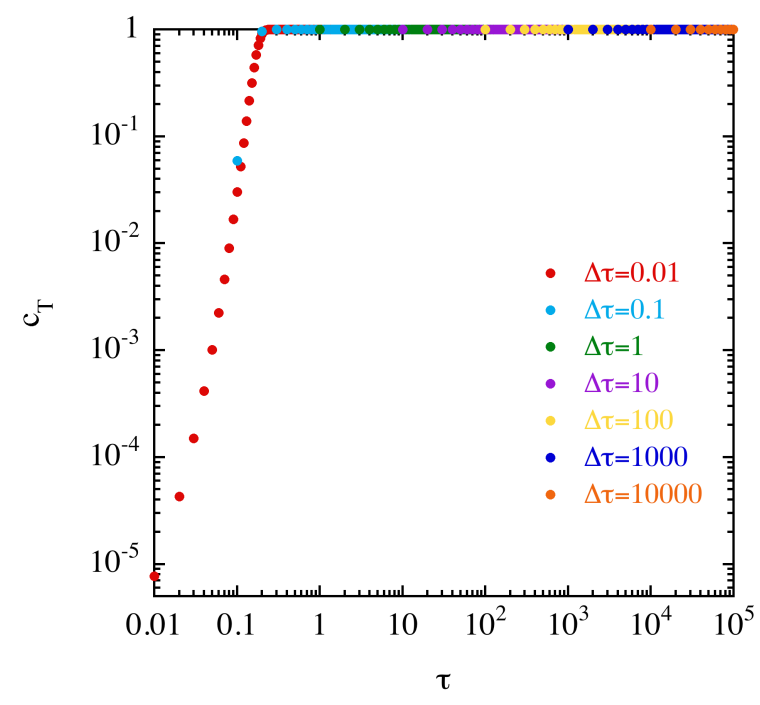

(b)

Figure 9. Evolution with $t$ of (a) $c_{L}$ and (b) $c_{T}$ for considering several fixed time increments. $c_{L}$ and $c_{T}$ results are considered at $x=0.5$. It is important to underline that with a constant time increment, Abaqus cannot control the error on $\mathrm{c}_{L}$, inducing under-estimated values (especially for $\Delta t=1$ and $10 \mathrm{~s}$ ), indicated by arrows.

The relative difference between the numerical (equation (5.5)) and the GOA (equation (2.14)) values for $\partial c_{T} / \partial c_{L}$ are evaluated by the following expression

$$
100\left|\frac{\left.\frac{\partial c_{T}}{\partial c_{L}}\right|_{\text {num }}-\left.\frac{\partial c_{T}}{\partial c_{L}}\right|_{G O A}}{\left.\frac{\partial c_{T}}{\partial c_{L}}\right|_{\text {num }}}\right|
$$

For all the fixed time increments $\Delta t$, these relative differences are plotted on Figure 10, as well as the correspond number of Newton-Raphson loops.

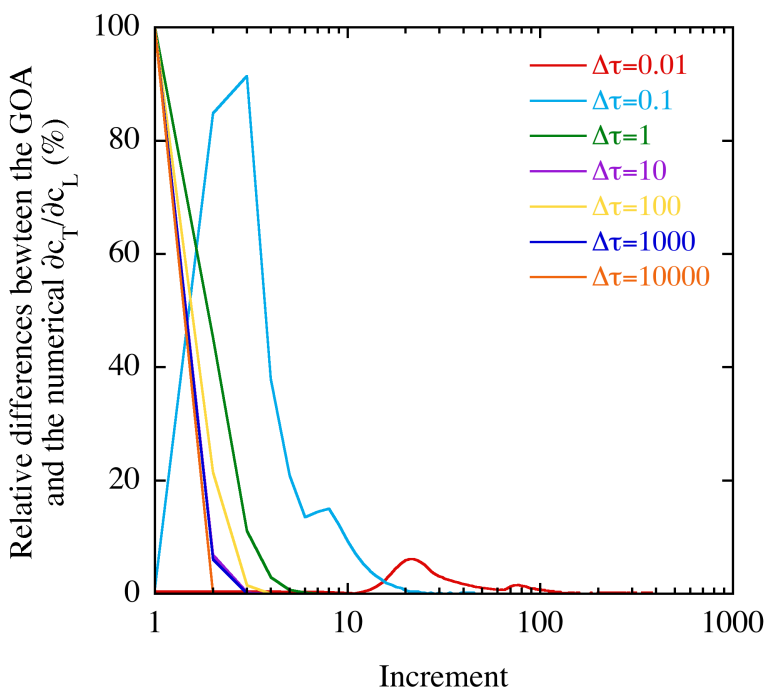

(a)

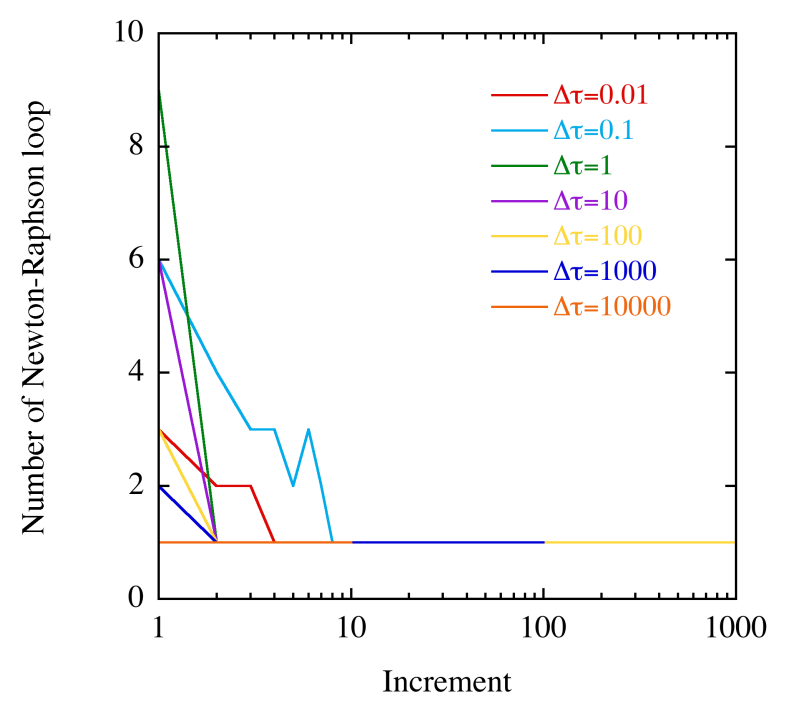

(b)

Figure 10. (a) Relative differences between $\partial c_{T} / \partial c_{L}$ computed numercially and using the GOA, and (b) number of Newton-Raphons loops needed to get a converged solution.

For there are no reference curves for these configurations, it is difficult to state about the accuracy of the computed $c_{L}$ and $c_{T}$ evolutions with $t$. However, for all the configurations, convergence was reached regardless on the fixed time increment $\Delta t$. For every configuration, results for the different fixed $\Delta t$ are on top of each other. From Figure 10, it can be seen that 
the $\partial c_{T} / \partial c_{L}$ value computed using the GOA fits perfectly to the numerical one, for -almostall of the configurations, but at the first increments. The smaller the difference between the numerical and the GOA $\partial c_{T} / \partial c_{L}$, the less Newton-Raphson loops are required to reach convergence. When $\Delta t$ is small, convergence is fast and the GOA $\partial c_{T} / \partial c_{L}$ is closed to the numerical one; for $\Delta t=10 \mathrm{~s}$, convergence for the first increments is slow and the GOA $\partial c_{T} / \partial c_{L}$ far from the numerical one: the GOA $\partial c_{T} / \partial c_{L}$ is consequently, in such a configuration, not accurate enough.

These results tend to indicate that the GOA and especially the computation of $\partial c_{T} / \partial c_{L}$, provides a good approximation of the real evolutions of $c_{L}, c_{T}$, and $d u d t$, but that such a conclusion must be mitigate by the value of $\Delta t$. This is due to the fact that the GOA $\partial c_{T} / \partial c_{L}$ at $t+\Delta t$ is dependent of the value of both $c_{T}(t)$ and $c_{L}(t)$ values: any error in the computations consequently has consequences on the $\partial c_{T} / \partial c_{L}$ computation.

\section{Conclusion}

The Generalized Oriani's Approximation (GOA) has been proposed in order to be able to provide Abaqus with the needed functions to reach convergence when modelling transport and transient trapping problems (and by extension, to provide an efficient approach to solve first order kinetic equations). The trapping processes considered are hydrogen in metals and water in polymers, described by first order differential equations.

In the present study, it has been shown that the GOA is able to provide an accurate estimation of the trapped impurities concentrations as soon as the time increment is small enough. An upper bound of the error between the GOA estimated trapped concentration and the theoretical one has been estimated to control the time increment in Finite Element computations. When applied on a given configuration, this estimation was shown to be very efficient in the case of both Carter \& Kibler and McNabb \& Foster equations, though for the latter, being unable to provide a correct enough upper-bound in the case of fast trapping and slow detrapping problems.

Last, it has been shown that the $d u d t$ parameters estimated by the GOA are indeed accurate enough to allow the convergence in Abaqus software of the considered transport and transient trapping problems. In the case of Carter \& Kibler trapping equation, the GOA provides the exact $d u d t$ value. As it has been proved, an inaccurate $d u d t$ parameter evaluation prevents the solver from converging.

Last, it is worth to underline that all the previous conclusions can be extended to any phenomenon that can modify hydrogen transport (as multi-trapping, stress-driven diffusion or Soret effect), as they only affect the $\theta_{L}$ temporal evolution at the considered point.

\section{Acknowledgments}

The author would like to thanks P. Gilormini, from PIMM, ENSAM, Paris, France, for fruitful discussions on the transient trapping and transport processes, and to have pointed out our attention of the Carter \& Kibler theoretical solution for the diffusion and trapping problem in polymers.

This work has been carried out within the framework of the EUROfusion consortium and has received funding from the Euratom research and training programme 2014-2018 and 20192020 under Grant Agreement No 633053. The views and opinions expressed herein do not necessarily reflect those of the European Commission. 
This work has been also carried out within the framework of the French Federation for Magnetic Fusion Studies (FR-FCM).

The authors thank R. Delaporte-Mathurin for his help in improving the article.

\section{Bibliography}

[1] Benannoune S, Charles Y, Mougenot J, Gaspérini M. Numerical simulation of the transient hydrogen trapping process using an analytical approximation of the McNabb and Foster equation. Int J Hydrog Energy 2018;43:9083-93. doi:10.1016/j.ijhydene.2018.03.179.

[2] Birnbaum HK, Sofronis P. Hydrogen-enhanced localized plasticity - a mechanism for hydrogen-related fracture. Mater Sci Eng A 1994;176:191-202. doi:10.1016/09215093(94)90975-X.

[3] Tabata T, Birnbaum HK. Direct observations of the effect of hydrogen on the behavior of dislocations in iron. Scripta Metall 1983;17:947-50. doi:10.1016/00369748(83)90268-5.

[4] Yagodzinskyy Y, Saukkonen T, Kilpelainen S, Tuomisto F, Hanninen H. Effect of hydrogen on plastic strain localization in single crystals of austenitic stainless steel. Scr Mater 2010;62:155-8. doi:10.1016/j.scriptamat.2009.10.005.

[5] Shen C-H, Springer GS. Effects of Moisture and Temperature on the Tensile Strength of Composite Materials:. J Compos Mater 1977;11:2-16. doi:10.1177/002199837701100102.

[6] Bernstein IM. The role of hydrogen in the embrittlement of iron and steel. Mater Sci Eng A 1970;6:1-19. doi:10.1016/0025-5416(70)90073-X.

[7] Dwivedi SK, Vishwakarma M. Hydrogen embrittlement in different materials: A review. Int J Hydrog Energy 2018;43:21603-16. doi:10.1016/j.ijhydene.2018.09.201.

[8] Gwak G, Kim M, Oh K, Kyoung S, Ferekh S, Ju H. Analyzing effects of volumetric expansion of uranium during hydrogen absorption. Int J Hydrog Energy 2016. doi:10.1016/j.ijhydene.2016.08.176.

[9] Ayadi S, Charles Y, Gaspérini M, Caron Lemaire I, Da Silva Botelho T. Effect of loading mode on blistering in iron submitted to plastic prestrain before hydrogen cathodic charging. Int J Hydrog Energy 2017;42:10555-67. doi:10.1016/j.ijhydene.2017.02.048.

[10] Quirós C, Mougenot J, Lombardi G, Redolfi M, Brinza O, Charles Y, et al. Blister formation and hydrogen retention in aluminium and beryllium: A modeling and experimental approach. Nucl Mat Ener 2017;12:1178-83. doi:10.1016/j.nme.2016.12.036.

[11] Ren XC, Zhou QJ, Shan GB, Chu W, Li JX, Su YJ, et al. A Nucleation Mechanism of Hydrogen Blister in Metals and Alloys. Metall Mater Trans A 2007;39:87-97. doi:10.1007/s11661-007-9391-3.

[12] Tiegel MC, Martin ML, Lehmberg AK, Deutges M, Borchers C, Kirchheim R. Crack and blister initiation and growth in purified iron due to hydrogen loading. Acta Mater 2016;115:24-34. doi:10.1016/j.actamat.2016.05.034.

[13] Castagnet S, Lainé E, D’Amore A, Acierno D, Grassia L. Analysis of the creep behavior of thin polymer films by finite elements simulation of micro-bubble inflation. AIP Conf Proc 2010;1255:152-4. doi:10.1063/1.3455561.

[14] Laiarinandrasana L, Morgeneyer TF, Proudhon H, N'guyen F, Maire E. Effect of Multiaxial Stress State on Morphology and Spatial Distribution of Voids in Deformed Semicrystalline Polymer Assessed by X-ray Tomography. Macromolecules 2012;45:4658-68. doi:10.1021/ma3005247. 
[15] Li J, Oudriss A, Metsue A, Bouhattate J, Feaugas X. Anisotropy of hydrogen diffusion in nickel single crystals: the effects of self-stress and hydrogen concentration on diffusion. Sci Rep 2017;7:45041. doi:10.1038/srep45041.

[16] Svoboda J, Fischer FD. Anisotropy of interstitial diffusion in bcc-crystals due to stress-induced unequal occupancies of different types of sites. Int J Solids Struct 2018;152-153:66-70. doi:10.1016/j.ijsolstr.2018.05.023.

[17] Zhou J, Lucas JP. Hygrothermal effects of epoxy resin. Part I: the nature of water in epoxy. Polymer 1999;40:5505-12. doi:10.1016/S0032-3861(98)00790-3.

[18] Sar BE, Fréour S, Célino A, Jacquemin F. Accounting for differential swelling in the multi-physics modeling of the diffusive behavior of a tubular polymer structure. J Compos Mater 2014;49:2375-87. doi:10.1177/0021998314546704.

[19] Kumnick AJ, Johnson HH. Deep trapping states for hydrogen in deformed iron 1980;28:33-9. doi:10.1016/0001-6160(80)90038-3.

[20] Choo WY, Lee J-Y. Thermal analysis of trapped hydrogen in pure iron. Met Trans A 1982;13:135-40. doi:10.1007/BF02642424.

[21] Gilormini P, Verdu J. On the role of hydrogen bonding on water absorption in polymers. Polymer 2018;142:164-96. doi:10.1016/j.polymer.2018.03.033.

[22] Vieth WR, Sladek KJ. A model for diffusion in a glassy polymer. Journal of Colloid Science 1965;20:1014-33. doi:10.1016/0095-8522(65)90071-1.

[23] McNabb A, Foster PK. A new analysis of the diffusion of hydrogen in iron and ferritic steels. Trans Metall Soc AIME 1963;227:618-27.

[24] Carter HG, Kibler KG. Langmuir-Type Model for Anomalous Moisture Diffusion In Composite Resins. J Compos Mater 1978;12:118-31.

doi:10.1177/002199837801200201.

[25] Drozdov AD, Christiansen JD, Gupta RK, Shah AP. Model for anomalous moisture diffusion through a polymer-clay nanocomposite. Journal of Polymer Science Part B: Polymer Physics 2003;41:476-92. doi:10.1002/polb.10393.

[26] Oriani RA. The diffusion and trapping of hydrogen in steel 1970;18:147-57. doi:10.1016/0001-6160(70)90078-7.

[27] Oh C-S, Kim YJ, Yoon KB. Coupled analysis of hydrogen transport using ABAQUS. J Solid Mech Mater Eng 2010;4:908-17. doi:10.1299/jmmp.4.908.

[28] Charles Y, Nguyen TH, Gaspérini M. Comparison of hydrogen transport through predeformed synthetic polycrystals and homogeneous samples by finite element analysis. Int J Hydrog Energy 2017;42:20336-50.

doi:10.1016/j.ijhydene.2017.06.016.

[29] Charles Y, Nguyen TH, Gaspérini M. FE simulation of the influence of plastic strain on hydrogen distribution during an U-bend test. Int J Mech Sci 2017;120:214-24. doi:10.1016/j.ijmecsci.2016.11.017.

[30] Moriconi C, Hénaff G, Halm D. Cohesive zone modeling of fatigue crack propagation assisted by gaseous hydrogen in metals. Int J Fatigue 2014;68:56-66. doi:10.1016/j.ijfatigue.2014.06.007.

[31] Alvaro A, Olden V, Akselsen OM. 3D cohesive modelling of hydrogen embrittlement in the heat affected zone of an X70 pipeline steel. Int J Hydrog Energy 2013;38:7539-49. doi:10.1016/j.ijhydene.2013.02.146.

[32] Kumar R, Mahajan DK. Hydrogen distribution in metallic polycrystals with deformation. J Mech Phys Solids 2020;135:103776. doi:10.1016/j.jmps.2019.103776.

[33] Dadfarnia M, Martin ML, Nagao A, Sofronis P, Robertson IM. Modeling hydrogen transport by dislocations. J Mech Phys Solids 2015;78:511-25. doi:10.1016/j.jmps.2015.03.002. 
[34] Benannoune S, Charles Y, Mougenot J, Gaspérini M, De Temmerman G. Multidimensional finite-element simulations of the diffusion and trapping of hydrogen in plasma-facing components including thermal expansion. Phys Scr 2020;T171:014011. doi:10.1088/1402-4896/ab4335.

[35] Charles Y, Gaspérini M, Ardon K, Ayadi S, Benannoune S, Mougenot J. Adaptation of hydrogen transport models at the polycrystal scale and application to the U-bend test. Procedia Struct Integrity 2018;13:896-901. doi:10.1016/j.prostr.2018.12.169.

[36] Charles Y, Gaspérini M, Fagnon N, Ardon K, Duhamel A. Finite element simulation of hydrogen transport during plastic bulging of iron submitted to gaseous hydrogen pressure. Eng Frac Mech 2019;218:106580. doi:10.1016/j.engfracmech.2019.106580.

[37] Benannoune S, Charles Y, Mougenot J, Gaspérini M, De Temmerman G. Numerical simulation by finite element modelling of diffusion and transient hydrogen trapping processes in plasma facing components. Nucl Mat Ener 2019;19:42-6. doi:10.1016/j.nme.2019.01.023.

[38] Díaz A, Alegre JM, Cuesta II, Zhang Z. Numerical study of hydrogen influence on void growth at low triaxialities considering transient effects. Int J Mech Sci 2019;164:105176. doi:10.1016/j.ijmecsci.2019.105176.

[39] Charles Y, Mougenot J, Gaspérini M. Effect of transient trapping on hydrogen transport near a blunting crack tip. Int J Hydrog Energy 2021;46:10995-1003. doi:10.1016/j.ijhydene.2020.12.155.

[40] Delaporte-Mathurin R, Hodille E, Mougenot J, Charles Y, De Temmerman G, Leblond F, et al. Influence of interface conditions on hydrogen transport studies. Nucl Fusion 2021;63:036038. doi:10.1088/1741-4326/abd95f.

[41] Derrien K, Gilormini P. Interaction between Stress and Diffusion in Polymers. Defect Diffus Forum 2006;258-260:447-52. doi:10.4028/www.scientific.net/DDF.258260.447.

[42] Li JCM, Oriani RA, Darken LS. The Thermodynamics of Stressed Solids. Z Phys Chem 1966;49:271-90. doi:10.1524/zpch.1966.49.3_5.271.

[43] Bogkris JO, Beck W, Genshaw MA, Subramanyan PK, Williams FS. The effect of stress on the chemical potential of hydrogen in iron and steel 1971;19:1209-18. doi:10.1016/0001-6160(71)90054-X.

[44] Espenson JH. Chemical Kinetics and Reaction Mechanisms. McGraw-Hill; 1995.

[45] Drexler A, Depover T, Leitner S, Verbeken K, Ecker W. Microstructural based hydrogen diffusion and trapping models applied to Fe-CX alloys. J Alloy Compd 2020;826:154057. doi:10.1016/j.jallcom.2020.154057.

[46] Hodille EA, Ferro Y, Fernandez N, Becquart CS, Angot T, Layet JM, et al. Study of hydrogen isotopes behavior in tungsten by a multi trapping macroscopic rate equation model. Phys Scr 2016;2016:014011. doi:10.1088/0031-8949/2016/T167/014011.

[47] Simulia. Abaqus User subroutines reference guide. Dassault Système; 2011.

[48] Krom AHM, Koers RWJ, Bakker AD. Hydrogen transport near a blunting crack tip. J Mech Phys Solids 1999;47:971-92. doi:10.1016/S0022-5096(98)00064-7.

[49] Moro I. Fragilisation par l"hydrogène gazeux d"un acier ferrito-perlitique de grade API X80. Institut Carnot CIRIMAT/ENSIACET, 2009. http://ethesis.inptoulouse.fr/archive/00001031/01/Moro.pdf

[50] Press WH, Teukolsky SA, Vetterling WT, Flannery BP. Numerical Recipes in Fortran 77. vol. 1. Cambridge University Press; 1992.

[51] odeint n.d. https://docs.scipy.org/doc/scipy/reference/tutorial/integrate.html (accessed April 2020). 


\section{Annex}

\section{A. Theoretical solution of the trapping differential equation}

1. McNabb \& Foster trapping equation

Let's consider the differential equation written on equation (2.6)b1

$$
\frac{\partial \theta_{T}}{\partial t}+\left(p+k \theta_{L}\right) \theta_{T}=k \theta_{L}
$$

The related homogeneous differential equation is thus

$$
\frac{\partial \theta_{T}^{H}}{\partial \tau}+\left(p+k \theta_{L}\right) \theta_{T}^{H}=0
$$

the solution of which being

$$
\theta_{T}^{H}=B \exp \left[-\int_{0}^{t}\left(p+k \theta_{L}\right) d u\right]
$$

where $B$ is a constant to be identified using the initial conditions.

The variation of parameters method allows to get a particular solution $\theta_{T}^{p}$ of the inhomogeneous differential equation by assuming that

$$
\theta_{T}^{p}=B(t) \exp \left[-\int_{t_{0}}^{t}\left(p+k \theta_{L}\right) d u\right]
$$

Inserting $\theta_{T}^{p}$ in the equation (A.1) leads to

$$
\frac{d B(t)}{d \tau} \exp \left[-\int_{t_{0}}^{t}\left(p+k \theta_{L}\right) d u\right]=k \theta_{L}
$$

and thus it is possible to get $B$

$$
B(t)=\int_{t_{0}}^{t}\left(k \theta_{L} \exp \left[\int_{t_{0}}^{v}\left(p+k \theta_{L}\right) d u\right]\right) d v
$$

as well as $\theta_{T}^{p}$

$$
\theta_{T}^{p}=\int_{t_{0}}^{t}\left(k \theta_{L} \exp \left[\int_{t_{0}}^{v}\left(p+k \theta_{L}\right) d u\right]\right) d v \exp \left[-\int_{t_{0}}^{t}\left(p+k \theta_{L}\right) d u\right]
$$

Last, the solution of equation (A.1) is so that $\theta_{T}=\theta_{T}^{H}+\theta_{T}^{p}$

$$
\theta_{T}=\left\{B+\int_{t_{0}}^{t}\left(k \theta_{L} \exp \left[\int_{t_{0}}^{v}\left(p+k \theta_{L}\right) d u\right]\right) d v\right\} \exp \left[-\int_{t_{0}}^{t}\left(p+k \theta_{L}\right) d u\right]
$$

Using the initial condition 


$$
\theta_{T}\left(t=t_{0}\right)=\theta_{T}^{0}
$$

the equation (A.8) can be rewritten as

$$
\theta_{T}=\left\{\theta_{T}^{0}+\int_{t_{0}}^{t}\left(k \theta_{L} \exp \left[\int_{t_{0}}^{v}\left(p+k \theta_{L}\right) d u\right]\right) d v\right\} \exp \left[-\int_{t_{0}}^{t}\left(p+k \theta_{L}\right) d u\right]
$$

Let's denotes $F$ as

$$
F(t)=\int\left(k \theta_{L}(v) \exp \left[\int_{t_{0}}^{v}\left(p+k \theta_{L}\right) d u\right]\right) d v
$$

Equation (A.11) is then rewritten as

$$
\theta_{T}=\left[\theta_{T}^{0}+F(t)-F\left(t_{0}\right)\right] \exp \left[-\int_{t_{0}}^{t}\left(p+k \theta_{L}\right) d u\right]
$$

$\theta_{T}$ can be separated in two: a transient part $\theta_{T}^{t r}$

$$
\theta_{T}^{t r}=\theta_{T}^{0} \exp \left[-\int_{t_{0}}^{t}\left(p+k \theta_{L}\right) d u\right]
$$

and a steady-state one $\theta_{T}^{\text {st }}$

$$
\theta_{T}^{s t}=\left[F(t)-F\left(t_{0}\right)\right] \exp \left[-\int_{t_{0}}^{t}\left(p+k \theta_{L}\right) d u\right]
$$

\section{Carter \& Kibler trapping equation}

From the previous resolution scheme, the general solution of equation (2.6)b2

$$
\frac{\partial \theta_{T}}{\partial t}+p \theta_{T}=k \theta_{L}
$$

can be found as well as being so that

$$
\theta_{T}=\left(\theta_{T}^{0}+F(t)-F\left(t_{0}\right)\right) \mathrm{e}^{-p\left(t-t_{0}\right)}
$$

with

$$
F(t)=\int k \theta_{L}(v) \mathrm{e}^{p\left(v-t_{0}\right)} d u
$$

The same separation $\theta_{T}=\theta_{T}^{t r}+\theta_{T}^{s t}$ can also be made

$$
\theta_{T}^{t r}=\theta_{T}^{0} \mathrm{e}^{-p\left(t-t_{0}\right)}
$$

and

$$
\theta_{T}^{s t}=\left[F(t)-F\left(t_{0}\right)\right] \mathrm{e}^{-p\left(t-t_{0}\right)}
$$

\section{B. Rewriting of the McNabb \& Foster theoretical solution}

From the Mean value theorem, there exist $c \in\left[v, t_{n+1}\right]$ so that 


$$
\int_{v}^{t_{n+1}} \theta_{T}(v) d u=\theta_{L}(c)\left(t_{n+1}-v\right)
$$

leading to

$$
\exp \left[-\int_{v}^{t_{n+1}}\left(p+k \theta_{L}\right) d u\right]=\exp \left[-\left(p+k \theta_{L}(c)\right)\left(t_{n+1}-v\right)\right]
$$

and

$$
k \theta_{L}(v) \exp \left[-\int_{v}^{t_{n+1}}\left(p+k \theta_{L}\right) d u\right]=k \theta_{L}(v) \exp \left[-\left(p+k \theta_{L}(c)\right)\left(t_{n+1}-v\right)\right]
$$

Furthermore, there exist $d \epsilon\left[v, t_{n+1}\right]$ so that

$$
\theta_{L}\left(t_{n+1}\right)-\theta_{L}(v)=\theta_{L}^{\prime}(d)\left(t_{n+1}-v\right)
$$

and equation (B.3) becomes

$$
\begin{gathered}
k \theta_{L}(v) \exp \left[-\int_{v}^{t_{n+1}}\left(p+k \theta_{L}\right) d u\right] \\
=k \theta_{L}\left(t_{n+1}\right) \exp \left[-\left(p+k \theta_{L}(c)\right)\left(t_{n+1}-v\right)\right] \\
-k \theta_{L}^{\prime}(d)\left(t_{n+1}-v\right) \exp \left[-\left(p+k \theta_{L}(c)\right)\left(t_{n+1}-v\right)\right]
\end{gathered}
$$

leading to

$$
\begin{gathered}
\int_{t_{n}}^{t_{n+1}} k \theta_{L}(v) \exp \left[-\int_{v}^{t_{n+1}}\left(p+k \theta_{L}\right) d u\right] d v \\
=\int_{t_{n}}^{t_{n+1}} k \theta_{L}\left(t_{n+1}\right) \exp \left[-\left(p+k \theta_{L}(c)\right)\left(t_{n+1}-v\right)\right] d v \\
-\int_{t_{n}}^{t_{n+1}} k \theta^{\prime}(d)\left(t_{n+1}-v\right) \exp \left[-\left(p+k \theta_{L}(c)\right)\left(t_{n+1}-v\right)\right] d v
\end{gathered}
$$

Equation (B.6) can be rewritten as

$$
\begin{gathered}
\int_{t_{n}}^{t_{n+1}} k \theta_{L}(v) \exp \left[-\int_{v}^{t_{n+1}}\left(p+k \theta_{L}\right) d u\right] d v \\
=\frac{k \theta_{L}\left(t_{n+1}\right)}{p+k \theta_{L}(c)}\left(1-e^{-\left[p+k \theta_{L}(c)\right] \Delta t}\right) \\
-k \theta_{L}^{\prime}(d)\left\lfloor\frac{1}{\left(p+k \theta_{L}(c)\right)^{2}}\left(1-\mathrm{e}^{-\left(p+k \theta_{L}(c)\right) \Delta t}\right)-\frac{\Delta t \mathrm{e}^{-\left(p+k \theta_{L}(c)\right) \Delta t}}{\left(p+k \theta_{L}(c)\right)}\right\rfloor
\end{gathered}
$$

When $\Delta t \rightarrow 0$, then equation (B.7) becomes 


$$
\begin{gathered}
\int_{t_{n}}^{t_{n+1}} k \theta_{L}(v) \exp \left[-\int_{v}^{t_{n+1}}\left(p+k \theta_{L}\right) d u\right] d v \\
\approx \frac{k \theta_{L}\left(t_{n+1}\right)}{p+k \theta_{L}\left(t_{n+1}\right)}\left(1-e^{-\left[p+k \theta_{L}\left(t_{n+1}\right)\right] \Delta t}\right)-k \theta_{L}^{\prime}\left(t_{n+1}\right) \frac{\Delta t^{2}}{2}
\end{gathered}
$$

with

$$
\mathrm{e}^{-\left(p+k \theta_{L}\left(t_{n+1}\right)\right) \Delta t} \approx 1-\left(p+k \theta_{L}\left(t_{n+1}\right)\right) \Delta t+\frac{\left(p+k \theta_{L}\left(t_{n+1}\right)\right)^{2}}{2} \Delta t^{2}
$$

\section{Rewriting of the Carter \& Kibler theoretical solution}

From the Mean value theorem, there exist $c \in\left[u, t_{n+1}\right]$ so that

$$
\theta_{L}\left(t_{n+1}\right)-\theta_{L}(u)=\theta_{L}^{\prime}(c)\left(t_{n+1}-u\right)
$$

leading to

$$
\theta_{L}\left(t_{n+1}\right) \mathrm{e}^{p\left(u-t_{n}\right)}-\theta_{L}(u) \mathrm{e}^{p\left(u-t_{n}\right)}=\theta^{\prime}{ }_{L}(c)\left(t_{n+1}-u\right) \mathrm{e}^{p\left(u-t_{n}\right)}
$$

and

$$
\begin{gathered}
\int_{t_{n}}^{t_{n+1}} \theta_{L}\left(t_{n+1}\right) \mathrm{e}^{p\left(u-t_{n}\right)} d u-\int_{t_{n}}^{t_{n+1}} \theta_{L}(u) \mathrm{e}^{p\left(u-t_{n}\right)} \\
=\theta^{\prime}{ }_{L}(c) \int_{t_{n}}^{t_{n+1}}\left(t_{n+1}-u\right) \mathrm{e}^{p\left(u-t_{n}\right)} d u
\end{gathered}
$$

Equation (C.3)'s right hand side can be rewritten as

$$
\int_{t_{n}}^{t_{n+1}}\left(t_{n+1}-u\right) \mathrm{e}^{p\left(u-t_{n}\right)} d u=\frac{1}{p^{2}}\left(\mathrm{e}^{p \Delta t}-1\right)-\frac{\Delta t}{p}
$$

leading to

$$
\begin{aligned}
& \frac{k}{p} \theta_{L}\left(t_{n+1}\right)\left(1-\mathrm{e}^{-p \Delta t}\right)-\int_{t_{n} k}^{t_{n+1}} k \theta_{L}(u) \mathrm{e}^{p\left(u-t_{n}\right)} d u \mathrm{e}^{-p \Delta t} \\
& =k \theta^{\prime}{ }_{L}(c)\left[\frac{1}{p^{2}}\left(1-\mathrm{e}^{-p \Delta t}\right)-\frac{\Delta t}{p} \mathrm{e}^{-p \Delta t}\right]
\end{aligned}
$$

It is worth noting that

1. when $p \rightarrow 0$ (or $\Delta t \rightarrow 0$ ), equation (C.5) becomes

$$
k \theta_{L}\left(t_{n+1}\right)-\int_{t_{n} k}^{t_{n+1}} k \theta_{L}(u) d u=k \theta^{\prime}{ }_{L}(c) \frac{\Delta t^{2}}{2}
$$


which is the classical right or left rectangular result for the approximation of $\int_{t_{n} k}^{t_{n+1}} k \theta_{L}(u) d u$.

2. Equation (C.5) corresponds to (B.7) when $\left|k \theta_{L}(t)\right| \ll p, \forall t$. 